\title{
Digital Technology Interventions for Risk Factor Modification in Patients With Cardiovascular Disease: Systematic Review and Meta-analysis
}

\author{
Adewale Samuel Akinosun ${ }^{1}$, BSc, MPH; Rob Polson ${ }^{2}$, MSc; Yohanca Diaz - Skeete ${ }^{3}$, BSc, MSc; Johannes Hendrikus \\ De Kock ${ }^{1}$, PhD; Lucia Carragher ${ }^{3}$, PhD; Stephen Leslie ${ }^{4}$, MBChB, PhD; Mark Grindle ${ }^{1}$, MLitt, PhD; Trish Gorely ${ }^{1}$, \\ BPhEd, MEd, PhD

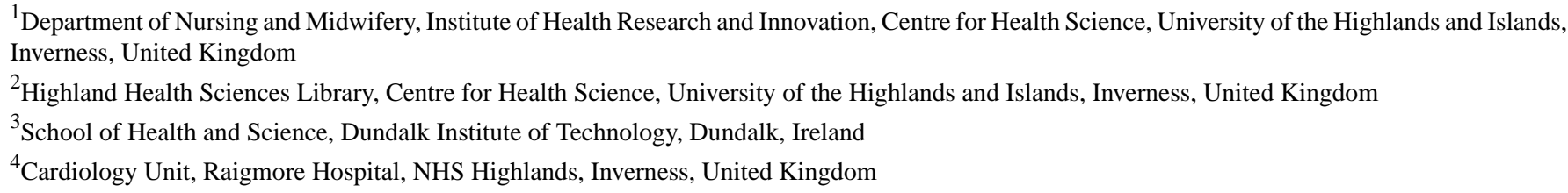

Corresponding Author:

Adewale Samuel Akinosun, BSc, MPH

Department of Nursing and Midwifery, Institute of Health Research and Innovation

Centre for Health Science

University of the Highlands and Islands

Inverness

United Kingdom

Phone: 4407727867360

Email: $\underline{18026171 @ u h i . a c . u k}$

\section{Abstract}

Background: Approximately 50\% of cardiovascular disease (CVD) cases are attributable to lifestyle risk factors. Despite widespread education, personal knowledge, and efficacy, many individuals fail to adequately modify these risk factors, even after a cardiovascular event. Digital technology interventions have been suggested as a viable equivalent and potential alternative to conventional cardiac rehabilitation care centers. However, little is known about the clinical effectiveness of these technologies in bringing about behavioral changes in patients with CVD at an individual level.

Objective: The aim of this study is to identify and measure the effectiveness of digital technology (eg, mobile phones, the internet, software applications, wearables, etc) interventions in randomized controlled trials (RCTs) and determine which behavior change constructs are effective at achieving risk factor modification in patients with CVD.

Methods: This study is a systematic review and meta-analysis of RCTs designed according to the PRISMA (Preferred Reporting Items for Systematic Reviews and Meta-analysis) statement standard. Mixed data from studies extracted from selected research databases and filtered for RCTs only were analyzed using quantitative methods. Outcome hypothesis testing was set at $95 \%$ CI and $P=.05$ for statistical significance.

Results: Digital interventions were delivered using devices such as cell phones, smartphones, personal computers, and wearables coupled with technologies such as the internet, SMS, software applications, and mobile sensors. Behavioral change constructs such as cognition, follow-up, goal setting, record keeping, perceived benefit, persuasion, socialization, personalization, rewards and incentives, support, and self-management were used. The meta-analyzed effect estimates (mean difference [MD]; standard mean difference $[\mathrm{SMD}]$; and risk ratio $[\mathrm{RR}]$ ) calculated for outcomes showed benefits in total cholesterol SMD at -0.29 [-0.44, $-0.15], P<.001$; high-density lipoprotein SMD at $-0.09[-0.19,0.00], P=.05$; low-density lipoprotein SMD at $-0.18[-0.33$, -0.04], $P=.01$; physical activity (PA) SMD at 0.23 [0.11, 0.36], $P<.001$; physical inactivity (sedentary) RR at 0.54 [0.39, 0.75$]$, $P<.001$; and diet (food intake) RR at 0.79 [0.66, 0.94], $P=.007$. Initial effect estimates showed no significant benefit in body mass index (BMI) MD at -0.37 [ $-1.20,0.46], P=.38$; diastolic blood pressure (BP) SMD at $-0.06[-0.20,0.08], P=.43$; systolic BP SMD at $-0.03[-0.18,0.13], P=.74$; Hemoglobin $A_{1 C}$ blood sugar $\left(\mathrm{HbA}_{1 \mathrm{c}}\right)$ RR at $1.04[0.40,2.70], P=.94 ;$ alcohol intake SMD at -0.16 [-1.43, 1.10], $P=.80$; smoking RR at 0.87 [0.67, 1.13], $P=.30$; and medication adherence RR at 1.10 [1.00, 1.22$], P=.06$. 
Conclusions: Digital interventions may improve healthy behavioral factors (PA, healthy diet, and medication adherence) and are even more potent when used to treat multiple behavioral outcomes (eg, medication adherence plus). However, they did not appear to reduce unhealthy behavioral factors (smoking, alcohol intake, and unhealthy diet) and clinical outcomes (BMI, triglycerides, diastolic and systolic $\mathrm{BP}$, and $\mathrm{HbA}_{1 \mathrm{c}}$ ).

(JMIR Mhealth Uhealth 2021;9(3):e21061) doi: 10.2196/21061

\section{KEYWORDS}

digital technologies; mHealth; eHealth; risk factors; cardiovascular diseases; telehealth; cardiac rehabilitation; behavior; systematic review; meta-analysis; mobile phone

\section{Introduction}

\section{Background}

Cardiovascular diseases (CVDs), including coronary heart disease (CHD), stroke, and peripheral vascular disease, remain one of the most common causes of early death and disability worldwide, with 17.9 million deaths and 422.7 million cases each year [1]. In 2017 alone, there were approximately 1.7 million inpatient episodes of CVDs in the United Kingdom [2]. This imposes a heavy burden on individuals and the society, accounting for $£ 19$ billion in public expense, 7.4 million disabilities, and 167,000,000 deaths in 2019 [1].

Although there are genetic, demographic, and environmental causes of CVDs [3], approximately 50\% of CVD risk is attributable to modifiable lifestyle factors such as obesity, diabetes, inactivity, and smoking [4]. However, despite widespread education and personal knowledge, many individuals fail to adequately modify these risk factors, even after a cardiovascular event with cardiac rehabilitation care center support [4]. Failure to address this challenge (ie, a change from cognitive insight to manifest action) results in patients remaining at a higher risk of future cardiovascular events with associated personal, social, and economic costs.

There could be several reasons for the challenges in the personalized management (ie, modification) of CVD and other chronic disease risk factors. These include care center accessibility and outpatient mobility and morbidity, comprehensibility, and retainability [5]. For these reasons, lifestyle risk factor management (particularly low physical activity [PA] and obesity) remains suboptimally addressed in CVD outpatients [6]. These show that although there is a modest success with rehabilitation care center interventions, the technical reach of this population-based approach is limited in its ability to bring about a significant sustainable change in exposed individuals [5].

To achieve a sustainable change, social construct strategies (eg, self-management, motivation, perceived benefits, etc) embedded in behavioral change interventions have shown health benefits in chronic disease risk factor management [7]. Their substantial contribution to changes in health behaviors suggests a worthy consideration in behavioral health interventions at the individual level. However, in the context of a population-based health behavior change in rehabilitation care centers, there are limitations in the ability of social constructs to make an individual cope sustainably with its strategies at the personal level [6].
The emergence of digital health technologies (eg, the internet, phone apps and devices, and wearable sensors for telemedicine, web browsing, emailing, text messaging, monitoring) in the health care sector [8], designed to manage and monitor chronic disease lifestyle factors, has shown potential in personalized chronic disease lifestyle factor modification [9]. This potential is based on evidence that healthy lifestyle factors are behavior-specific, measurable, and modifiable [10]. Due to the commercial drive and attributed qualities, many of these technologies and devices have been continually used in cardiac rehabilitation care centers, and even instead of care [11,12]. However, despite their popularity and potential, these technologies lack evidence summary of secondary prevention of clinically relevant outcomes, which result from behavior change in CVD outpatients, especially at a personalized level $[12,13]$.

\section{Objectives}

The primary objective of this systematic review is to identify and measure the effectiveness of digital technology (eg, mobile phones, the internet, software applications, wearables, etc) interventions in randomized controlled trials (RCTs) and determine which behavior change constructs were effective at achieving risk factor modification among patients with CVD.

\section{Methods}

\section{Study Design}

This study is a systematic literature review and meta-analysis of RCTs designed in line with the PRISMA (Preferred Reporting Items for Systematic Reviews and Meta-analysis) statement standard [14]. The protocol was registered with PROSPERO protocol ID CRD42019139801 [15].

\section{Inclusion Criteria for Considering Studies in This Review}

\section{Types of Studies}

RCTs of digital interventions with a minimum of 4 weeks of intervention follow-up period were considered. Publications in English from 2000 to September 2019 were included.

\section{Types of Participants}

The considered studies focused on an adult population $(\geq 18$ years) with a minimum of 30 participants in the intervention study. 


\section{Types of Interventions}

The considered intervention types were digital intervention only versus usual care or digital intervention plus usual care versus usual care. The studies were required to be based on a well-defined CVD risk factor modification measurement function and intention to modify health behavior in outpatients diagnosed or treated for CVD only or with comorbidities using a named digital device.

\section{Types of Outcome Measures}

Clinical and behavioral outcomes were measured at baseline and endpoint. All outcome hypothesis testing was set at $95 \%$ $\mathrm{CI}$ and a 2-tailed $P$ value of .05 level of statistical significance. Behavioral outcomes included PA (physical inactivity [PI], sedentary lifestyle), food intake (diet), smoking, alcohol intake, and medication adherence. Clinical outcomes included BMI, cholesterol levels (total cholesterol [TC], high-density lipoproteins [HDLs], low-density lipoproteins [LDLs], and triglycerides [TGs]), blood pressure (diastolic BP and systolic $\mathrm{BP})$, and blood sugar levels $\left(\mathrm{HbA}_{1 \mathrm{c}}\right)$, which are measures of obesity, hypercholesterolemia, hypertension, and diabetes, respectively.

\section{Exclusion Criteria}

Studies related to non-RCTs, nondigital interventions, and journal papers not published in English were excluded. In addition, studies whose populations shared modifiable risk factors with CVD but were not diagnosed with a named CVD; studies with nonclinical or nonbehavioral outcomes, such as studies with genetic outcomes and studies directly measuring hospital or staff service efficiency, etc; and healthy population or intensive care studies were excluded.

No analysis was conducted on data of outcomes from subgroups (endpoint to endpoint) within the population of the included studies.

\section{Search Methods for Identification of Studies}

A single 6S pyramid systematic literature search strategy was developed (Table S3 in the Multimedia Appendix 1). This was run on Ovid Medline and Ovid Embase, Web of Science (Core Collection), Scopus, Cochrane Library, and on the following databases in EBSCOHost: CINAHL, Psych Info, Health Source, Open Dissertation, Psych Article, and Business Source Elite. Filters were used to narrow searches to studies using RCT methodology and those written in the English language and from the year 2000 onward. The year limit was applied, in line with the World Health Organization's release of the document on the approach to digital health strategies [16], as the start of the mass availability and use of digital technologies, making pre-2000 literature less relevant.

Two independent reviewers (AS and RP) were involved in a thorough search strategy build-up and study extraction to identify potentially relevant publications. References and citations were also searched. Where an abstract did not provide sufficient precision to meet the selection prerequisite, the article was reserved for full-text review. Relevant articles retrieved for full-text reviews were independently evaluated (AS and MG). The consensus to include or exclude a trial was reached based on study design, method, population demography, intervention mechanism, and study outcomes.

\section{Data Collection and Analysis}

\section{Data Collection}

The PRISMA search protocol [14] was followed, with all extracted data subsequently managed using the Mendeley Desktop reference manager software (Elsevier). Publication search outcomes were imported in .ris format into the Mendeley Desktop and partitioned based on the search database source. Imported publications were autochecked for duplicates using the software, and a further manual, independent duplication check was carried out (AS and YD). Publication papers were title-read, abstract-read, and full-text read based on the inclusion and exclusion preselection criteria. Selected journal papers were read for data synthesis and analysis.

\section{Data Extraction and Management}

Data were extracted into a preset Excel (Microsoft Corporation) worksheet. The data extraction process was performed independently (AS) using predetermined variables and then validated accordingly (MG). Data extracted included population demographics (mean age, sex, size, and CVD diagnosis) description of the study (authors, year of publication, country, intervention acronym, digital device, intervention type, trial protocol registration, design, and duration), behavioral change context (change technique and risk factors), and clinical study outcomes (outcome measures, outcome units, mean baseline measurements, mean outcome measurements, $P$ values, and SDs). Authors of studies with insufficient or missing outcome data were contacted for further information.

\section{Data Analysis}

All extracted data from the selected studies were analyzed (Table 1) using Review Manager 5.3 (The Cochrane Collaboration). An assessment of the risk of bias (Table 2) was carried out by 2 researchers, AS and YD, using the modified Cochrane Collaboration AUB KQ1 Risk of Bias Assessment Tool, Review Manager 5.3 (The Cochrane Collaboration) with assessment result validation by an external independent researcher. Bias quality was assessed as high, low, or unclear for individual elements from 6 items: selection, performance, attrition, reporting, proportion, outcome, and treatment efficacy. Where the attrition bias risk is high, there is more likely to be a high treatment efficacy bias except where the basis for participant dropout is a medical reason, relocation, or death. Quality assessment items were evaluated by an external assessor to validate the initial scales judged by the author. Controversial evaluation differences were discussed, and consensus was reached before the final documentation. The risk of bias across studies was assessed for each analyzed outcome for publication bias reporting. Results were generated with meta-analysis data for each outcome and are presented in the Results section.

The review authors considered the variations in outcome measurement across studies by applying appropriate statistical methods (fixed effect and random effect) using the Inverse-Variance and Mantel-Haenszel (DerSimonian and Laird) models to generate meta-analytic estimates of treatment effect 
using the Review Manager 5.3 software. Differences in effects were examined by comparing digital with usual care. The weighted mean difference (MD) or standardized mean difference (SMD) was calculated for continuous data using the inverse variance statistical method. Relative risks were calculated for dichotomous data using the Mantel-Haenszel statistical method (The Cochrane Collaboration). Provision for variations among the included studies was made using the random effect meta-analysis model in analyzing all included studies. The heterogeneity statistic $\mathrm{I}^{2}$ was calculated to describe the percentage of variation among the studies. Hypothesis testing was set at a 2-tailed 0.05 level of significance and a 95\% CI. No analysis was conducted on the data of outcomes from subgroups within the population of the included studies.

Sensitivity analyses were proportionately conducted on outcomes to check the cumulative effects of the publication year, participant size, efficacy, and category of intervention (risk factors and digital intervention) on statistical significance. For food intake, studies with interventions targeting healthy diet and studies targeting unhealthy diet were analyzed separately to provide a clearer insight into treatment effects. Studies with treatment for medication adherence were analyzed separately for (1) other risk factor treatments plus medication adherence using the SMS text messaging intervention only, (2) medication adherence treatment only (with no other risk factor) using the SMS text messaging intervention alone, (3) other risk factor treatment plus medication adherence treatment using non-SMS text messaging intervention, and (4) medication adherence treatment with SMS text messaging intervention only. The results are presented and discussed in the Results and Discussion sections, respectively.

Table 1. Summary of meta-analysis results ${ }^{\mathrm{a}}$.

\begin{tabular}{|c|c|c|c|c|c|}
\hline Outcomes or subgroups & $\begin{array}{l}\text { Number of studies } \\
(\mathrm{N}=25), \mathrm{n}(\%)\end{array}$ & Participants & Statistical methods & Effect estimates $(95 \% \mathrm{CI})$ & $P$ value \\
\hline BMI & $10(40)$ & 2558 & $\mathrm{MD}^{\mathrm{b}}(\mathrm{IV}$, random, 95\% CI) & $-0.37(-1.20$ to 0.46$)$ & .38 \\
\hline Total cholesterol & $9(36)$ & 1783 & $\mathrm{SMD}^{\mathrm{c}}(\mathrm{IV}$, random, $95 \% \mathrm{CI})$ & $-0.29(-0.44$ to -0.15$)$ & $<.001$ \\
\hline High-density lipoprotein & $9(36)$ & 1783 & SMD (IV, random, 95\% CI) & $-0.09(-0.19$ to 0.00$)$ & .05 \\
\hline Low-density lipoprotein & $12(48)$ & 3431 & SMD (IV, random, 95\% CI) & $-0.18(-0.33$ to -0.04$)$ & .01 \\
\hline Triglycerides & $8(32)$ & 1660 & SMD (IV, random, 95\% CI) & $-0.07(-0.24$ to 0.11$)$ & .28 \\
\hline Diastolic $\mathrm{BP}^{\mathrm{d}}$ & $11(44)$ & 2460 & SMD (IV, random, 95\% CI) & $-0.06(-0.20$ to 0.08$)$ & .43 \\
\hline Systolic BP & $12(48)$ & 3283 & SMD (IV, random, 95\% CI) & $-0.03(-0.18$ to 0.13$)$ & .74 \\
\hline Physical activity & $14(56)$ & 3015 & SMD (IV, random, 95\% CI) & $0.23(0.11$ to 0.36$)$ & $<.001$ \\
\hline Alcohol consumption & $4(16)$ & 651 & SMD (IV, random, 95\% CI) & $-0.16(-1.43$ to 1.10$)$ & .80 \\
\hline Blood sugar, $\mathrm{HbA}_{1 \mathrm{c}}{ }^{\mathrm{e}}$ & $2(8)$ & 380 & $\mathrm{RR}^{\mathrm{f}}(\mathrm{M}-\mathrm{H}$, random, $95 \% \mathrm{CI})$ & $1.04(0.40$ to 2.70$)$ & .94 \\
\hline Physical inactivity & $4(16)$ & 1054 & RR (M-H, random, 95\% CI) & $0.54(0.39$ to 0.75$)$ & $<.001$ \\
\hline Food intake (diet) & $6(24)$ & 716 & $\mathrm{RR}(\mathrm{M}-\mathrm{H}$, random, $95 \% \mathrm{CI})$ & $0.79(0.66$ to 0.94$)$ & .007 \\
\hline Health diet & $3(12)$ & 173 & $\mathrm{RR}(\mathrm{M}-\mathrm{H}$, random, $95 \% \mathrm{CI})$ & $0.70(0.55$ to 0.89$)$ & .004 \\
\hline Unhealthy diet & $3(12)$ & 185 & RR (M-H, random, 95\% CI) & $0.90(0.68$ to 1.19$)$ & .47 \\
\hline Smoking & $11(44)$ & 2916 & $\mathrm{RR}(\mathrm{M}-\mathrm{H}$, random, $95 \% \mathrm{CI})$ & $0.87(0.67$ to 1.13$)$ & .30 \\
\hline Medication adherence & $11(44)$ & 2710 & $\mathrm{RR}(\mathrm{M}-\mathrm{H}$, random, $95 \% \mathrm{CI})$ & $1.10(1.00$ to 1.22$)$ & .06 \\
\hline $\begin{array}{l}\text { Medication adherence (multiple } \\
\text { treatment) }\end{array}$ & $5(20)$ & 758 & $\mathrm{RR}(\mathrm{M}-\mathrm{H}$, random, $95 \% \mathrm{CI})$ & 1.07 (1.01 to 1.14$)$ & .02 \\
\hline
\end{tabular}

${ }^{\mathrm{a}}$ Summary of analyzed data.

${ }^{\mathrm{b}} \mathrm{MD}$ : mean difference.

${ }^{\mathrm{c}} \mathrm{SMD}$ : standard mean difference.

${ }^{\mathrm{d}} \mathrm{BP}$ : blood pressure.

${ }^{\mathrm{e}} \mathrm{HbA}_{1 \mathrm{c}}$ : Hemoglobin $\mathrm{A}_{1 \mathrm{c}}$ blood sugar.

${ }^{\mathrm{f}} \mathrm{RR}$ : risk ratio 
Table 2. Risk of bias in included studies ${ }^{\mathrm{a}}$.

\begin{tabular}{|c|c|c|c|c|c|c|c|c|c|}
\hline \multirow[t]{2}{*}{ Study (reference) } & \multicolumn{2}{|c|}{ Selection bias } & \multirow{2}{*}{$\begin{array}{l}\text { Perfor- } \\
\text { mance bias } \\
\text { Blinding of } \\
\text { participants } \\
\text { and person- } \\
\text { nel }\end{array}$} & \multirow{2}{*}{$\begin{array}{l}\text { Detection } \\
\text { bias } \\
\text { Blinding of } \\
\text { outcome } \\
\text { assessment }\end{array}$} & \multirow{2}{*}{$\begin{array}{l}\text { Attrition } \\
\text { bias } \\
\text { Incomplete } \\
\text { outcome } \\
\text { data }\end{array}$} & \multirow{2}{*}{$\begin{array}{l}\text { Proportion } \\
\text { bias } \\
\text { Groups bal- } \\
\text { anced at } \\
\text { baseline }\end{array}$} & \multirow{2}{*}{$\begin{array}{l}\text { Outcome } \\
\text { bias } \\
\text { Groups re- } \\
\text { ceived } \\
\text { same inter- } \\
\text { vention }\end{array}$} & \multirow{2}{*}{$\begin{array}{l}\text { Reportin } \\
\text { bias } \\
\text { Selective } \\
\text { reporting }\end{array}$} & \multirow{2}{*}{$\begin{array}{l}\text { Treatmen } \\
\text { efficacy } \\
\text { Intention- } \\
\text { to-treat } \\
\text { analysis }\end{array}$} \\
\hline & $\begin{array}{l}\text { Random } \\
\text { sequence } \\
\text { generation }\end{array}$ & $\begin{array}{l}\text { Allocation } \\
\text { conceal- } \\
\text { ment }\end{array}$ & & & & & & & \\
\hline Akhu-Z et al, 2016 [17] & Low & Unclear & Unclear & Low & High & Low & Low & Low & High \\
\hline Chow et al, 2015 [18] & Low & Low & Low & Low & High & Low & High & Low & High \\
\hline Dale et al, 2015 [19] & Low & Low & Low & High & Low & High & High & Low & Low \\
\hline Devi et al, 2014 [20] & Low & Low & Low & High & High & Low & Low & High & Unclear \\
\hline Frederix et al, 2015 [21] & Low & Low & Low & Low & High & Low & Low & Low & Unclear \\
\hline Hawkes et al, 2012 [22] & Low & High & Low & Low & High & Low & Low & High & Unclear \\
\hline Johnston et al, 2016 [23] & Low & Unclear & Unclear & Unclear & High & High & High & High & Unclear \\
\hline Kamal et al, 2015 [24] & Low & Low & Low & Low & High & Low & High & Low & High \\
\hline Khonsari et al, 2015 [25] & Low & Unclear & High & Unclear & Low & Low & High & Low & Low \\
\hline Kraal et al, 2014 [26] & Low & Low & Unclear & Unclear & Low & Low & High & Low & Low \\
\hline Lear et al, 2014 [27] & Low & Low & Low & Low & High & Low & Low & Low & Low \\
\hline Maddison et al, 2014 [28] & Low & Low & Low & Low & High & Low & High & Low & Unclear \\
\hline Ogren et al, 2018 [29] & Low & Low & High & Unclear & Low & Low & Low & Unclear & High \\
\hline Pandey et al, 2014 [30] & Unclear & Unclear & Low & Low & Unclear & Low & Low & Unclear & Unclear \\
\hline Park et al, 2013 [31] & Low & Low & High & Low & Low & Unclear & Low & Low & Low \\
\hline Quilici et al, 2012 [32] & Low & Low & Unclear & Unclear & High & Low & Low & Low & Unclear \\
\hline Redfern et al, 2009 [33] & Low & Low & Low & Low & Low & Low & Low & Low & Low \\
\hline Reid et al, 2011 [34] & Low & Low & Low & Low & High & Low & Low & Low & Low \\
\hline Southard et al, 2003 [35] & Low & Low & Low & Low & Low & Low & Low & Low & Low \\
\hline Tiede et al, 2017 [36] & Low & Low & High & Unclear & High & High & Low & Low & High \\
\hline Vale et al, 2002 [37] & Low & High & High & Low & High & Low & Low & High & Low \\
\hline Vemooij et al, 2012 [38] & Low & Low & Unclear & Unclear & Low & Low & High & Low & Low \\
\hline Wan et al, 2016 [39] & Low & Low & Low & Low & High & Low & Low & Low & Low \\
\hline Widmer et al, 2017 [40] & Low & Low & Low & Low & Low & High & High & Low & Unclear \\
\hline Zheng et al, 2019 [41] & Low & High & Low & Low & Low & Low & High & Low & Low \\
\hline
\end{tabular}

${ }^{\mathrm{a}}$ Risk of bias: review authors' judgments about each risk of bias item for the included studies.

\section{Results}

\section{Search Results}

The search retrieved 1626 papers with the auto-removal of 326 duplicates. A total of 35 papers remained after applying inclusion and exclusion criteria. Ten papers were excluded because they were systematic literature reviews but not RCTs.
A final count of 25 papers was considered for the review: 12 from the database searches and 13 from references, citations, and gray literature (Figure 1). The included studies are listed in the table of included studies (Table S4 in Multimedia Appendix 1), and excluded studies are listed in the table of excluded studies (Table S5 in Multimedia Appendix 1) with reasons. 
Figure 1. Database search flowchart. RCT: randomized controlled trial.

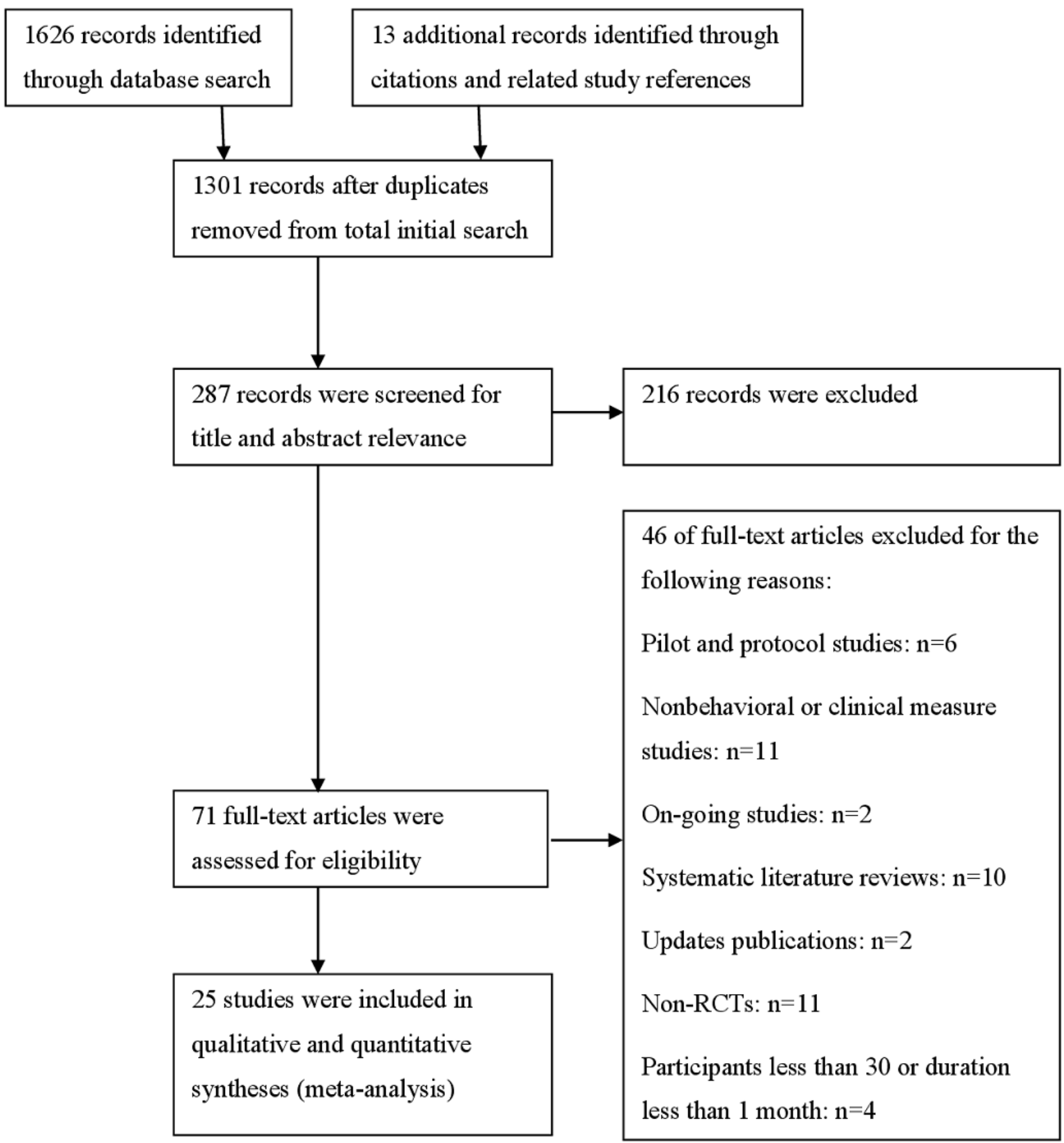

\section{Study Characteristics}

Studies are described by their common characteristics, which include population demography, digital technologies and brands, intervention mechanisms and behavioral change constructs, types of CVD, and general characteristics. Table S6 (Multimedia Appendix 2) provides a detailed summary of the reviewed studies.

\section{Population Demography}

The included studies had a total participant count of 5,779 at baseline, with a mean age of 60.03 years (SD 2.73) and a male proportion of $75.22 \%$ (4347/5779). A geographical analysis of the included studies identified evenly distributed locations of studies on a global scale, with countries spanning Europe (5 studies), Middle East (2 studies), Asia (3 studies), Northern America (6 studies), Scandinavia (2 studies), Australasia (6 studies), and the United Kingdom (1 study).

\section{Digital Technologies and Brands}

Cardiovascular digital interventions were delivered using devices such as cell phones, smartphones, personal computers (laptops and desktops), and wearables. Technologies included the internet, software applications, and mobile sensors. Intervention device brand names such as Personal Health Assistant, PHA, FIT@Home, HeartLinks, SUPPORT, 
SMS4Stroke, ProActive Heart, Text4Heart, CHAT, CardioFit, HEART, ActivateYourHeart, MEMS, vCRP, COACH, CHOICE, and TBHC were recorded.

\section{Intervention Mechanisms and Behavioral Change Constructs}

Intervention mechanisms (ie, the digital strategy plus behavioral construct) were based on online support, telerehabilitation, telemonitoring, and online coaching. The interventions included major behavioral change constructs such as cognition, follow-up, goal setting, record keeping, perceived benefit, persuasion, social engagement (virtual), personalization (or customization), rewards and incentives, support, and self-management.

CVD types: Diagnosed CVDs included CHD, coronary artery disease, myocardial infarction, acute coronary syndrome, angina, atherosclerosis, heart failure, transient ischemic attack, and stroke. Four studies [17,27,35,42] were not specific about CVD diagnosis in the study population.

\section{General Characteristics}

Study follow-up ranged from 1 to 4 (9 studies), 6 (12 studies), 12 ( 3 studies), and 24 months ( 1 study), with 6 months being the most frequent duration of the follow-up period for interventions. No data on outcomes from subgroups within the populations of the included studies were considered in the analysis.

The main units of outcome measurements were $\mathrm{kg} / \mathrm{m}^{2}$ (BMI), $\mathrm{mg} / \mathrm{dL}$, and $\mathrm{mmol} / \mathrm{L}$ (TC, HDL, low-density lipoprotein [LDL], and TGs, mmHg (diastolic blood pressure [DBP] and systolic blood pressure [SBP]), min/week (PA and PI), percentage, \% (Hemoglobin $\mathrm{A}_{1 \mathrm{C}}$ blood sugar- $-\mathrm{HbA}_{1 \mathrm{c}}$, alcohol, smoking, and food intake), and Morisky Medication Adherence Scale for Medication Adherence 8. In the treatment context, all the intervention studies had been administered either as digital intervention versus usual care (15 studies) or digital intervention and usual care versus usual care (10 studies).

\section{Synthesis of Results}

The use of digital intervention compared with usual care significantly modified all CVD risk factors except BMI, TG, DBP, SBP, $\mathrm{HbA}_{1 \mathrm{c}}$, alcohol intake, smoking, and medication adherence. A detailed summary of these findings is presented in Table 1.

\section{Summary of Results}

Effect estimates (MD, SMD, and RR) were significant and in favor of digital interventions for TC, HDL, LDL, PA, PI, and food intake.

\section{Clinical Outcomes}

The BMI outcome (Figure 2) reported MD was estimated at -0.37 ( $95 \% \mathrm{CI}-1.20$ to $0.46, P=.38$ ). The TC outcome (Figure 3) reported an SMD estimated at $-0.29(95 \% \mathrm{CI}-0.44$ to -0.15 , $P<.001$ ). The HDL outcome (Figure 4) reported an SMD estimated at -0.09 (95\% CI -0.19 to $0.00, P=.05)$. The LDL outcome (Figure 5) reported an SMD estimated at $-0.18(95 \%$ CI -0.33 to $-0.04, P=.01$ ). The TG outcome (Figure 6$)$ reported an SMD estimated at $-0.10(95 \% \mathrm{CI}-0.28$ to 0.08$), P=.28$. Diastolic and systolic BP outcomes (Figures 7 and 8) reported SMDs estimated at $-0.06(95 \% \mathrm{CI}-0.20$ to $0.08, P=.43)$ and -0.03 (95\% CI -0.18 to $0.13, P=.74$ ), respectively. The $\mathrm{HbA}_{1 \mathrm{c}}$ outcome (Figure 9) reported an RR estimated at 1.04 (95\% CI 0.40 to $2.70, P=.94)$. A summary of the clinical outcome findings is presented in Figures 2-9.

Figure 2. Outcomes of the examined studies for BMI.

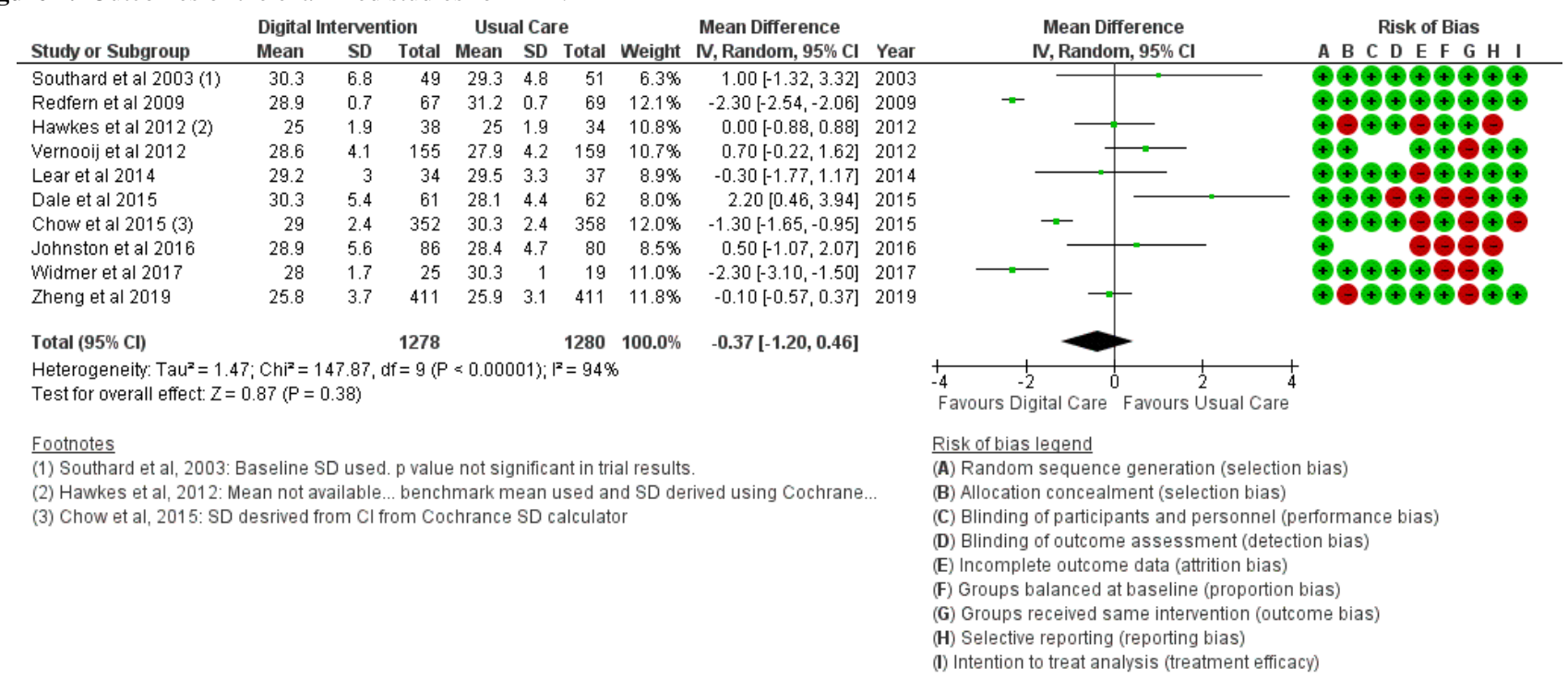


Figure 3. Outcomes of the examined studies for total cholesterol.

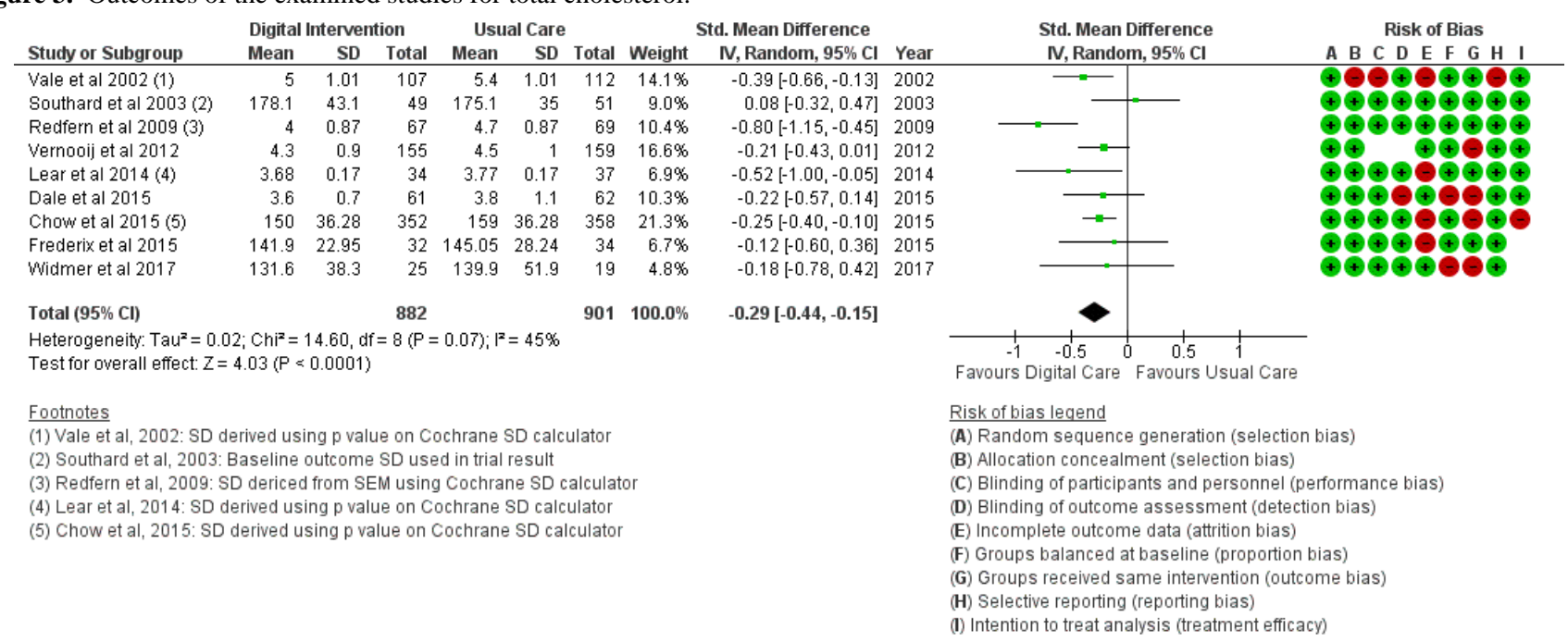

Figure 4. Outcomes of the examined studies for high-density lipoprotein.

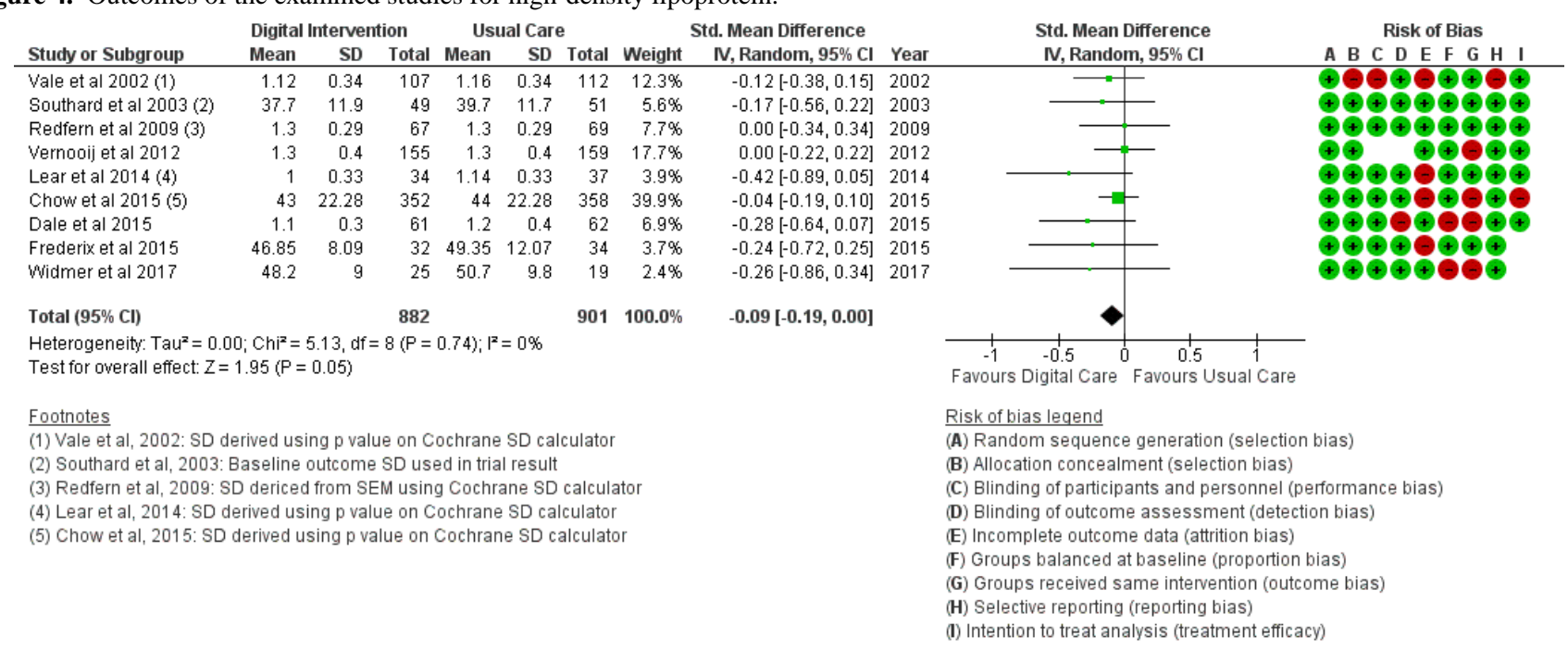

Figure 5. Outcomes of the examined studies for low-density lipoprotein.

\begin{tabular}{|c|c|c|c|c|c|c|c|c|c|c|c|c|}
\hline \multirow[b]{2}{*}{ Study or Subgroup } & \multicolumn{3}{|c|}{ Digital Intervention } & \multicolumn{3}{|c|}{ Usual Care } & \multicolumn{3}{|c|}{ Std. Mean Difference } & \multirow{2}{*}{\multicolumn{2}{|c|}{$\begin{array}{c}\text { Std. Mean Difference } \\
\text { IV, Random, } 95 \% \mathrm{Cl}\end{array}$}} & \multirow{2}{*}{$\begin{array}{c}\text { Risk of Bias } \\
\text { A B C DEF G }\end{array}$} \\
\hline & Mean & SD & Total & Mean & SD & Total & Weight & IV, Random, 95\% Cl & Year & & & \\
\hline Vale et al 2002 (1) & 3.11 & 0.92 & 107 & 3.57 & 0.92 & 112 & $9.2 \%$ & $-0.50[-0.77,-0.23]$ & 2002 & & & 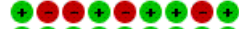 \\
\hline Southard et al 2003 (2) & 103.4 & 36.5 & 49 & 107.9 & 25.4 & 51 & $6.8 \%$ & $-0.14[-0.54,0.25]$ & 2003 & & & \\
\hline Redfern et al 2009 (3) & 2 & 0.74 & 67 & 2.4 & 0.74 & 69 & $7.7 \%$ & $-0.54[-0.88,-0.20]$ & 2009 & & & \\
\hline Vernooij et al 2012 & 2.3 & 0.7 & 155 & 2.6 & 0.9 & 159 & $10.2 \%$ & $-0.37[-0.59,-0.15]$ & 2012 & & & \\
\hline Lear et al 2014 & 1.79 & 0.36 & 34 & 1.99 & 0.36 & 37 & $5.5 \%$ & $-0.55[-1.02,-0.07]$ & 2014 & & & \\
\hline Chow et al 2015 (4) & 79 & 32.37 & 352 & 84 & 32.37 & 358 & $11.8 \%$ & $-0.15[-0.30,-0.01]$ & 2015 & & & \\
\hline Dale et al 2015 & 1.7 & 0.6 & 61 & 1.9 & 0.8 & 62 & $7.4 \%$ & $-0.28[-0.64,0.07]$ & 2015 & & & \\
\hline Frederix et al 2015 & 74.13 & 17.83 & 32 & 73.2 & 23.55 & 34 & $5.4 \%$ & $0.04[-0.44,0.53]$ & 2015 & & & \\
\hline Johnston et al 2016 & 3.9 & 1.2 & 86 & 3.3 & 0.9 & 80 & $8.3 \%$ & $0.56[0.25,0.87]$ & 2016 & & & \\
\hline Widmer et al 2017 & 63.2 & 35.7 & 25 & 59 & 47.3 & 19 & $4.1 \%$ & $0.10[-0.50,0.70]$ & 2017 & & & \\
\hline Ogren et al 2018 (5) & 2.2 & 3.31 & 320 & 2.5 & 3.31 & 340 & $11.7 \%$ & $-0.09[-0.24,0.06]$ & 2018 & & & \\
\hline Zheng et al 2019 & 93.6 & 27.7 & 411 & 99.3 & 30.8 & 411 & $12.0 \%$ & $-0.19[-0.33,-0.06]$ & 2019 & & & \\
\hline Total $(95 \% \mathrm{Cl})$ & & & 1699 & & & 1732 & $100.0 \%$ & $-0.18[-0.33,-0.04]$ & & & & \\
\hline \multicolumn{10}{|c|}{$\begin{array}{l}\text { Heterogeneity: } \text { Tau }^{2}=0.04 ; \mathrm{Chi}^{2}=40.02, \mathrm{df}=11(\mathrm{P}<0.0001) ; \mathrm{I}^{2}=73 \% \\
\text { Test for overall effect: } Z=2.50(P=0.01)\end{array}$} & $\begin{array}{lcl}-1 & -0.5 & 0 \\
\text { Favours } & \text { Digital Care }\end{array}$ & $\begin{array}{lr}0 & 0.5 \\
\text { Favours U }\end{array}$ & \\
\hline \multicolumn{10}{|c|}{$\begin{array}{l}\text { Footnotes } \\
\text { (1) Vale et al, 2002: SD derived using p value on Cochrane SD calculator } \\
\text { (2) Southard et al, 2003: Baseline outcome SD used in trial result } \\
\text { (3) Redfern et al, 2009: SD dericed from SEM using Cochrane SD calculator } \\
\text { (4) Chow et al, 2015: SD derived using p value on Cochrane SD calculator } \\
\text { (5) Ogren et al, 2018: SD derived using p value on Cochrane SD calculator }\end{array}$} & \multicolumn{3}{|c|}{$\begin{array}{l}\text { Risk of bias legend } \\
\text { (A) Random sequence generation (selection bias) } \\
\text { (B) Allocation concealment (selection bias) } \\
\text { (C) Blinding of participants and personnel (performance bias) } \\
\text { (D) Blinding of outcome assessment (detection bias) } \\
\text { (E) Incomplete outcome data (attrition bias) } \\
\text { (F) Groups balanced at baseline (proportion bias) } \\
\text { (G) Groups received same intervention (outcome bias) } \\
\text { (H) Selective reporting (reporting bias) } \\
\text { (I) Intention to treat analysis (treatment efficacy) }\end{array}$} \\
\hline
\end{tabular}


Figure 6. Outcomes of the examined studies for triglycerides.

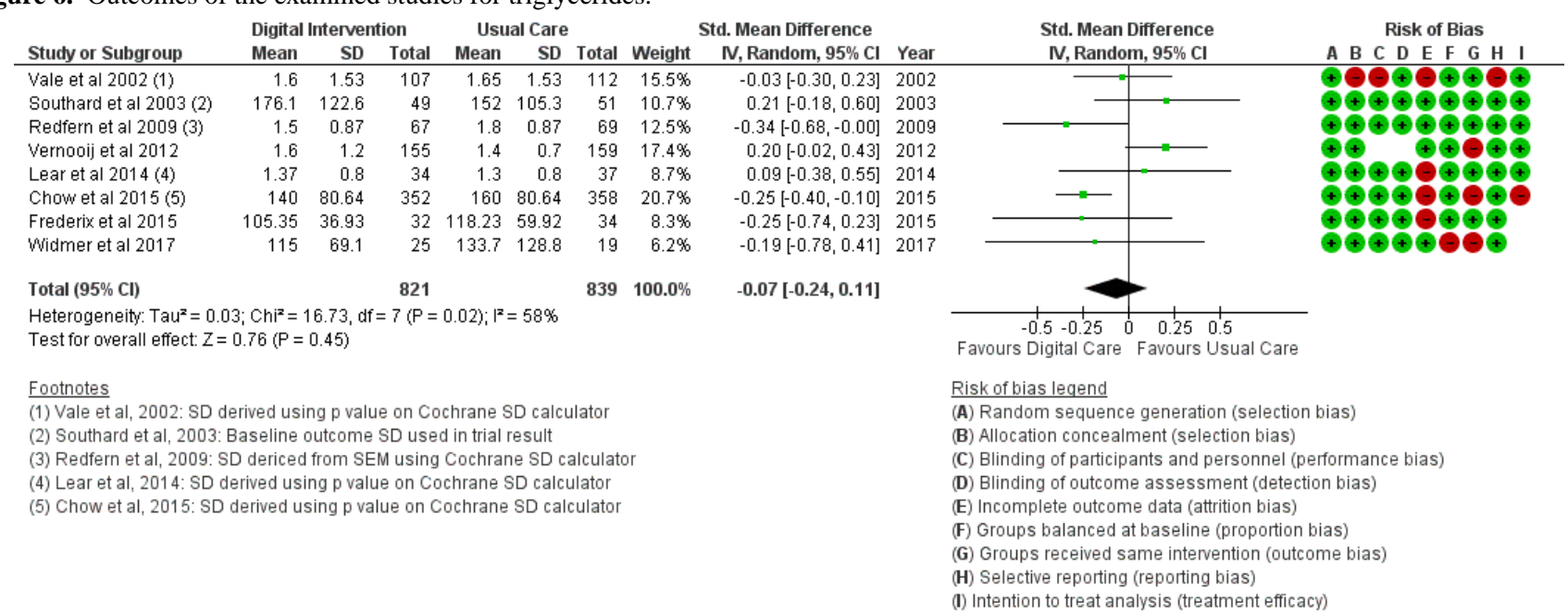

Figure 7. Outcomes of the examined studies for diastolic blood pressure.

\begin{tabular}{|c|c|c|c|c|c|c|c|c|c|c|c|c|}
\hline \multirow[b]{2}{*}{ Study or Subgroup } & \multicolumn{3}{|c|}{ Digital Intervention } & \multicolumn{3}{|c|}{ Usual Care } & \multicolumn{3}{|c|}{ Std. Mean Difference } & \multirow{2}{*}{\multicolumn{2}{|c|}{$\begin{array}{c}\text { Std. Mean Difference } \\
\text { N, Random, } 95 \% \mathrm{Cl}\end{array}$}} & Risk of Bias \\
\hline & Mean & SD & Total & Mean & SD & Total & Weight & IV, Random, $95 \% \mathrm{Cl}$ & Year & & & A B C D E F G H I \\
\hline Southard et al 2003 (1) & 72.5 & 10 & 49 & 71.8 & 9.2 & 51 & $7.5 \%$ & $0.07[-0.32,0.46]$ & 2003 & & & 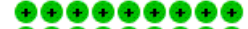 \\
\hline Redfern et al 2009 & 77.7 & 10.84 & 67 & 82.5 & 10.84 & 69 & $8.8 \%$ & $-0.44[-0.78,-0.10]$ & 2009 & & & $\hookrightarrow \odot \odot \odot \odot \odot \oplus \odot \odot$ \\
\hline Vernooij et al 2012 & 80 & 9 & 155 & 80 & 10 & 159 & $12.6 \%$ & $0.00[-0.22,0.22]$ & 2012 & & & \\
\hline Lear et al 2014 (2) & 76 & 14.25 & 34 & 77 & 14.25 & 37 & $6.1 \%$ & $-0.07[-0.54,0.40]$ & 2014 & & & \\
\hline Devi et al 2014 & 69 & 9.57 & 39 & 68.52 & 9.16 & 42 & $6.6 \%$ & $0.05[-0.39,0.49]$ & 2014 & & & \\
\hline Kamal et al 2015 & 77.9 & 9.16 & 83 & 80.5 & 9.16 & 79 & $9.7 \%$ & $-0.28[-0.59,0.03]$ & 2015 & & & \\
\hline Dale et al 2015 & 79 & 11 & 57 & 79 & 10 & 59 & $8.2 \%$ & $0.00[-0.36,0.36]$ & 2015 & & & \\
\hline Chow et al 2015 & 82.9 & 7.5 & 352 & 82.9 & 7.4 & 358 & $15.3 \%$ & $0.00[-0.15,0.15]$ & 2015 & & - & \\
\hline Frederix et al 2015 & 89 & 15 & 32 & 97 & 24 & 34 & $5.7 \%$ & $-0.39[-0.88,0.10]$ & 2015 & & & \\
\hline Widmer et al 2017 & 66.2 & 10.2 & 25 & 69.2 & 13.2 & 19 & $4.2 \%$ & $-0.25[-0.85,0.34]$ & 2017 & & & \\
\hline Ogren et al 2018 (3) & 75.3 & 13.22 & 320 & 71.9 & 13.22 & 340 & $15.1 \%$ & $0.26[0.10,0.41]$ & 2018 & & & \\
\hline Total $(95 \% \mathrm{Cl})$ & & & 1213 & & & 1247 & $100.0 \%$ & $-0.06[-0.20,0.08]$ & & & & \\
\hline \multicolumn{10}{|c|}{$\begin{array}{l}\text { Heterogeneity: } \text { Tau }^{2}=0.03 ; \mathrm{Chi}^{2}=23.74, \mathrm{df}=10(P=0.008) ;\left.\right|^{2}=58 \% \\
\text { Test for overall effect: } Z=0.78(P=0.43)\end{array}$} & $\begin{array}{ccc} & 1 & + \\
-1 & -0.5 & 0 \\
\text { Favours } & \text { Digital Care }\end{array}$ & Favours U & \\
\hline \multicolumn{10}{|c|}{$\begin{array}{l}\text { Footnotes } \\
\text { (1) Southard et al, 2003: Baseline outcome SD used in trial result. } \\
\text { (2) Lear et al, 2014: SD derived using p value on Cochrane SD calculator } \\
\text { (3) Ogren et al, 2018: SD derived using p value on Cochrane SD calculator }\end{array}$} & \multicolumn{3}{|c|}{$\begin{array}{l}\text { Risk of bias leqend } \\
\text { (A) Random sequence generation (selection bias) } \\
\text { (B) Allocation concealment (selection bias) } \\
\text { (C) Blinding of participants and personnel (performance bias) } \\
\text { (D) Blinding of outcome assessment (detection bias) } \\
\text { (E) Incomplete outcome data (attrition bias) } \\
\text { (F) Groups balanced at baseline (proportion bias) } \\
\text { (G) Groups received same intervention (outcome bias) } \\
\text { (H) Selective reporting (reporting bias) } \\
\text { (I) Intention to treat analysis (treatment efficacy) }\end{array}$} \\
\hline
\end{tabular}

Figure 8. Outcomes of the examined studies for systolic blood pressure.

\begin{tabular}{|c|c|c|c|c|c|c|c|c|c|c|c|}
\hline \multirow[b]{2}{*}{ Study or Subgroup } & \multicolumn{3}{|c|}{ Digital Intervention } & \multicolumn{3}{|c|}{ Usual Care } & \multicolumn{3}{|c|}{ Std. Mean Difference } & \multirow{2}{*}{$\begin{array}{c}\text { Std. Mean Difference } \\
\text { IV, Random, } 95 \% \mathrm{Cl}\end{array}$} & Risk of Bias \\
\hline & Mean & SD & Total & Mean & SD & Total & Weight & N, Random, $95 \% \mathrm{Cl}$ & Year & & A B C D E F G H I \\
\hline Southard et al 2003 (1) & 129.4 & 17.5 & 49 & 128.8 & 19.8 & 51 & $7.2 \%$ & $0.03[-0.36,0.42]$ & 2003 & & 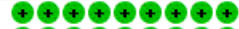 \\
\hline Redfern et al 2009 & 131.6 & 17.55 & 67 & 143.9 & 17.55 & 69 & $8.0 \%$ & $-0.70[-1.04,-0.35]$ & 2009 & & 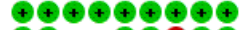 \\
\hline Vernooij et al 2012 & 137 & 18 & 155 & 140 & 19 & 159 & $10.4 \%$ & $-0.16[-0.38,0.06]$ & 2012 & & \\
\hline Lear et al 2014 (2) & 126 & 24.5 & 34 & 114 & 24.5 & 37 & $6.0 \%$ & $0.48[0.01,0.96]$ & 2014 & & \\
\hline Devi et al 2014 & 130.8 & 14.7 & 40 & 128.55 & 14.88 & 42 & $6.5 \%$ & $0.15[-0.28,0.58]$ & 2014 & & \\
\hline Chow et al 2015 & 128.8 & 12.3 & 352 & 128.7 & 12.2 & 358 & $11.8 \%$ & $0.01[-0.14,0.16]$ & 2015 & & \\
\hline Frederix et al 2015 & 135 & 24 & 32 & 128 & 22 & 34 & $5.8 \%$ & $0.30[-0.18,0.79]$ & 2015 & & \\
\hline Dale et al 2015 & 136 & 20 & 57 & 135 & 16 & 59 & $7.7 \%$ & $0.05[-0.31,0.42]$ & 2015 & & \\
\hline Johnston et al 2016 & 127.3 & 14.6 & 85 & 127.1 & 17.9 & 77 & $8.7 \%$ & $0.01[-0.30,0.32]$ & 2016 & & \\
\hline Widmer et al 2017 & 114.8 & 13.5 & 25 & 129.7 & 22.5 & 19 & $4.3 \%$ & $-0.82[-1.44,-0.19]$ & 2017 & & \\
\hline Ogren et al 2018 (3) & 128.1 & 23.75 & 320 & 122 & 23.75 & 340 & $11.7 \%$ & $0.26[0.10,0.41]$ & 2018 & $\rightarrow$ & \\
\hline Zheng et al 2019 & 127.6 & 14.6 & 411 & 129.4 & 15.7 & 411 & $11.9 \%$ & $-0.12[-0.26,0.02]$ & 2019 & & \\
\hline Total $(95 \% \mathrm{Cl})$ & & & 1627 & & & 1656 & $100.0 \%$ & $-0.03[-0.18,0.13]$ & & & \\
\hline \multicolumn{10}{|c|}{$\begin{array}{l}\text { Heterogeneity: } \operatorname{Tau}^{2}=0.05 ; \mathrm{Chi}^{2}=43.91, \mathrm{df}=11(\mathrm{P}<0.00001) ; \mathrm{I}^{2}=75 \% \\
\text { Test for overall effect: } Z=0.34(\mathrm{P}=0.74)\end{array}$} & $\begin{array}{ccccc}-1 & -0.5 & 0 & 0.5 & 1 \\
& & 0.5 & 1 \\
\text { Favours Digital Care } & \text { Favours Usual Care }\end{array}$ & \\
\hline \multicolumn{10}{|c|}{$\begin{array}{l}\text { Footnotes } \\
\text { (1) Southard et al, 2003: Baseline outcome SD used in trial result. } \\
\text { (2) Lear et al, 2014: SD derived using p value on Cochrane SD calculator } \\
\text { (3) Ogren et al, 2018: SD derived using p value on Cochrane SD calculator }\end{array}$} & \multicolumn{2}{|c|}{$\begin{array}{l}\text { Risk of bias legend } \\
\text { (A) Random sequence generation (selection bias) } \\
\text { (B) Allocation concealment (selection bias) } \\
\text { (C) Blinding of participants and personnel (performance bias) } \\
\text { (D) Blinding of outcome assessment (detection bias) } \\
\text { (E) Incomplete outcome data (attrition bias) } \\
\text { (F) Groups balanced at baseline (proportion bias) } \\
\text { (G) Groups received same intervention (outcome bias) } \\
\text { (H) Selective reporting (reporting bias) } \\
\text { (I) Intention to treat analysis (treatment efficacy) }\end{array}$} \\
\hline
\end{tabular}


Figure 9. Outcomes of the examined studies for blood sugar $\mathrm{HbA}_{1 \mathrm{c}}$.

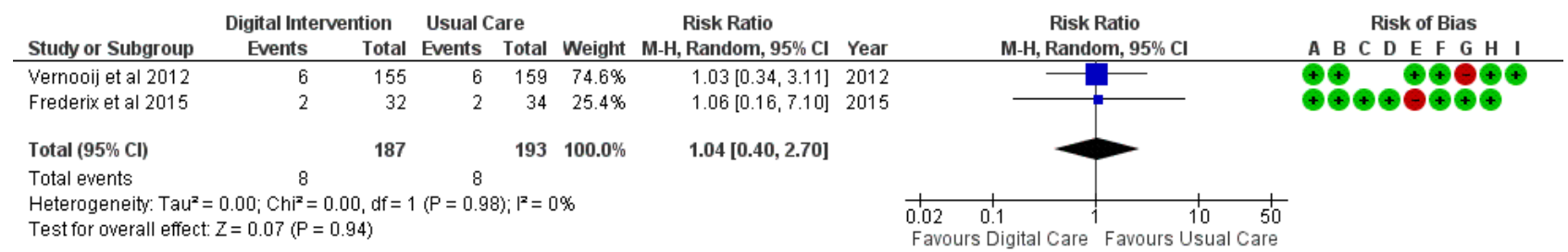

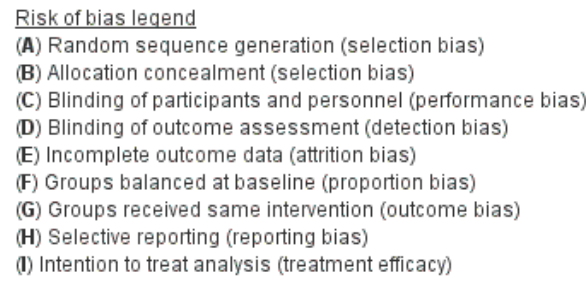

\section{Behavioral Outcomes}

The PA outcome (Figure 10) reported an SMD estimated at 0.23 (95\% CI 0.11 to $0.36, P<.001$ ). PI (sedentary) in Figure 11 reported an RR estimated at 0.54 (95\% CI 0.39 to 0.75 , $P<.001$ ). Diet (food intake) in Figure 12 reported an RR estimated at 0.79 ( 0.66 to $0.94, P=.007)$. Further analysis was conducted as healthy diet targeted (Figure 13) treatment and unhealthy diet targeted (Figure 14) treatment; this is reported in the AdditionalAnalysis section. The alcohol intake outcome (Figure 15) reported an SMD estimated at -0.16 (95\% CI -1.43 to $1.10, P=.80)$. Smoking and medication adherence outcomes (Figures 16 and 17) reported RR estimated at 0.87 (95\% CI 0.67 to $1.13, P=.30)$, and 1.10 (95\% CI 1.00 to $1.22, P=.06$ ), respectively. A summary of the behavioral outcome findings is presented in Figures 10-21.

Figure 10. Outcomes of the examined studies for physical activity.

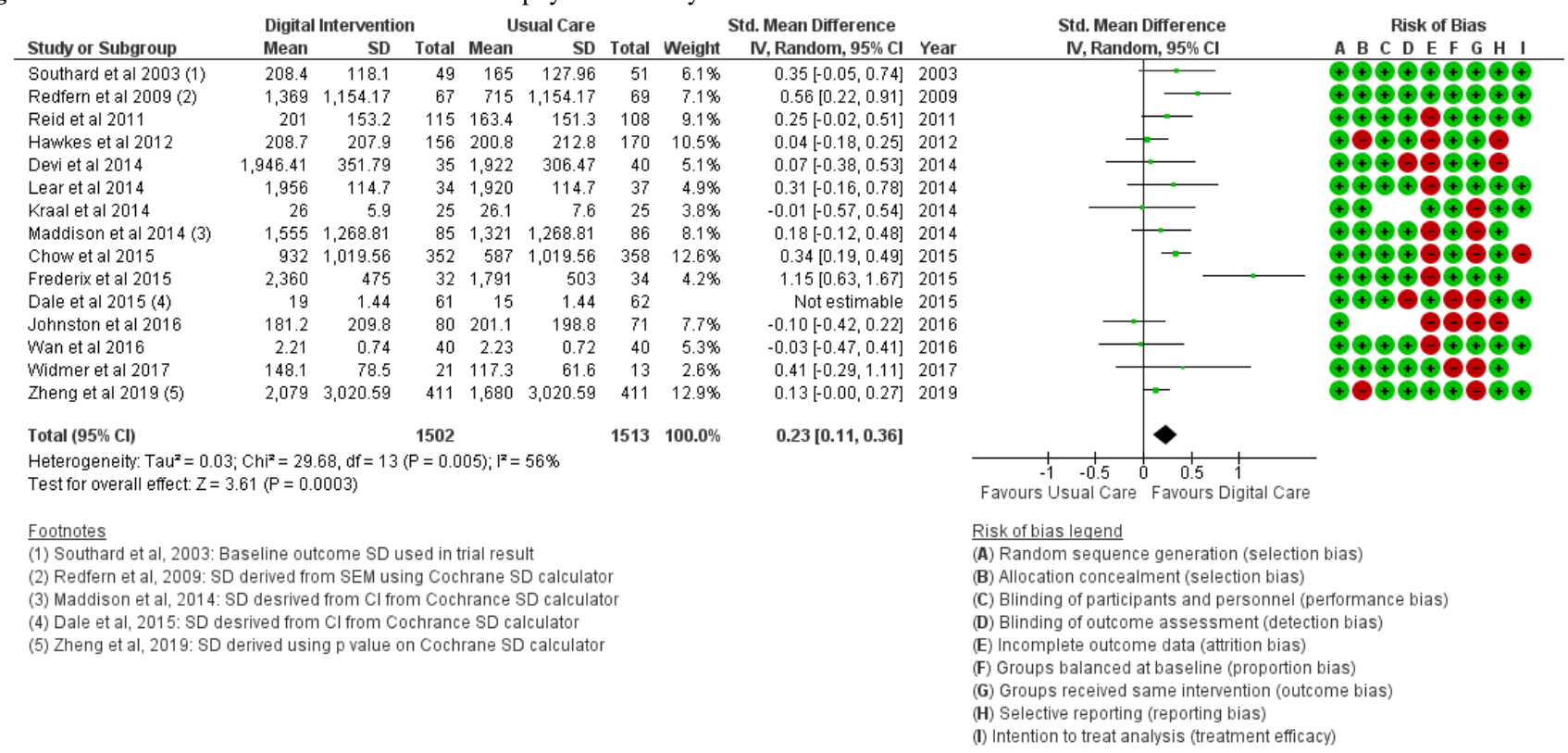


Figure 11. Outcomes of the examined studies for physical inactivity.

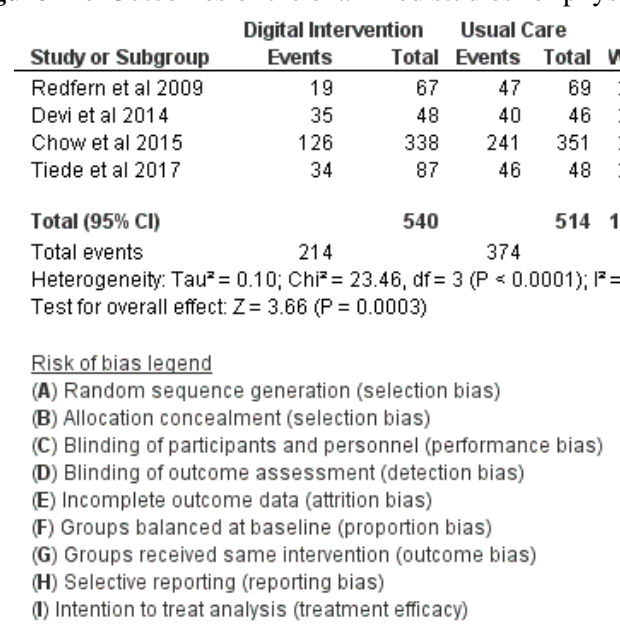

Risk Ratio

Risk Ratio

Risk of Bias

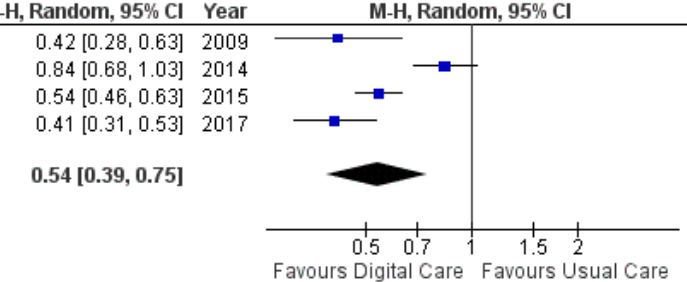

(I) Intention to treat analysis (treatment efficacy)

Figure 12. Outcomes of the examined studies for food intake.

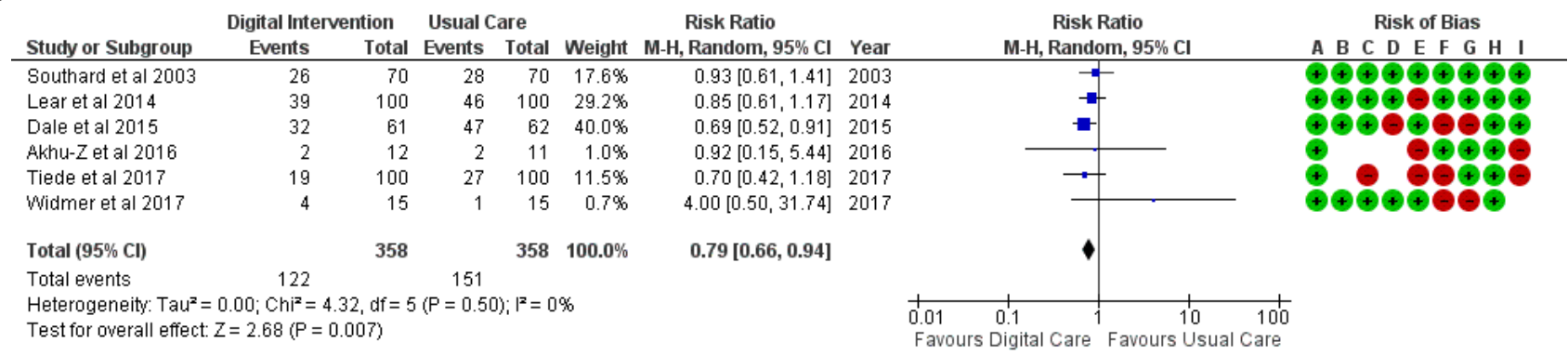

Risk of bias legend

(A) Random sequence generation (selection bias)

(B) Allocation concealment (selection bias)

(C) Blinding of participants and personnel (performance bias)

(D) Blinding of outcome assessment (detection bias)

(E) Incomplete outcome data (attrition bias)

(F) Groups balanced at baseline (proportion bias)

(G) Groups received same intervention (outcome bias)

(H) Selective reporting (reporting bias)

(I) Intention to treat analysis (treatment efficacy)

Figure 13. Outcomes of the examined studies for healthy diet.

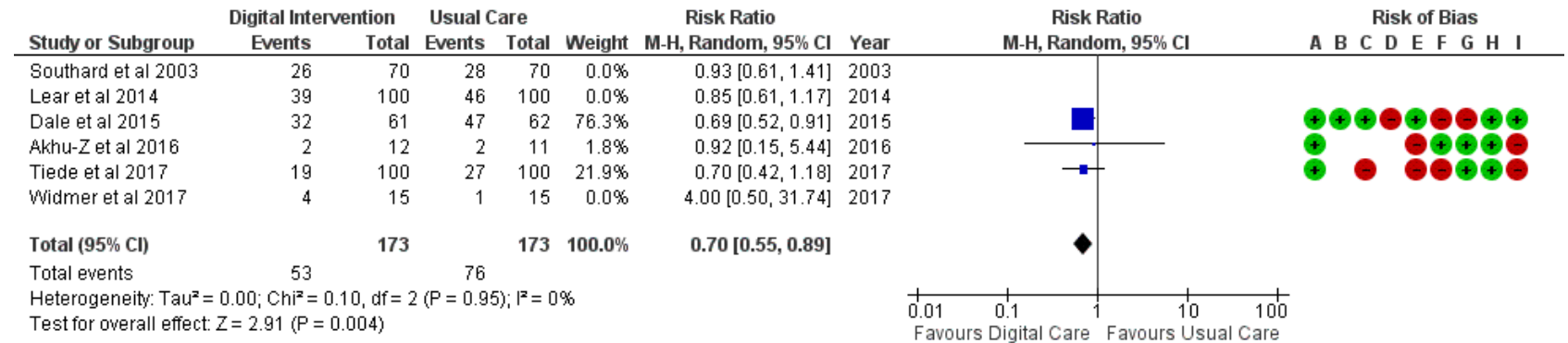

Risk of bias legend

(A) Random sequence generation (selection bias)

(B) Allocation concealment (selection bias)

(C) Blinding of participants and personnel (performance bias)

(D) Blinding of outcome assessment (detection bias)

(E) Incomplete outcome data (attrition bias)

(F) Groups balanced at baseline (proportion bias)

(G) Groups received same intervention (outcome bias)

(H) Selective reporting (reporting bias)

(I) Intention to treat analysis (treatment efficacy) 
Figure 14. Outcomes of the examined studies for unhealthy food intake.

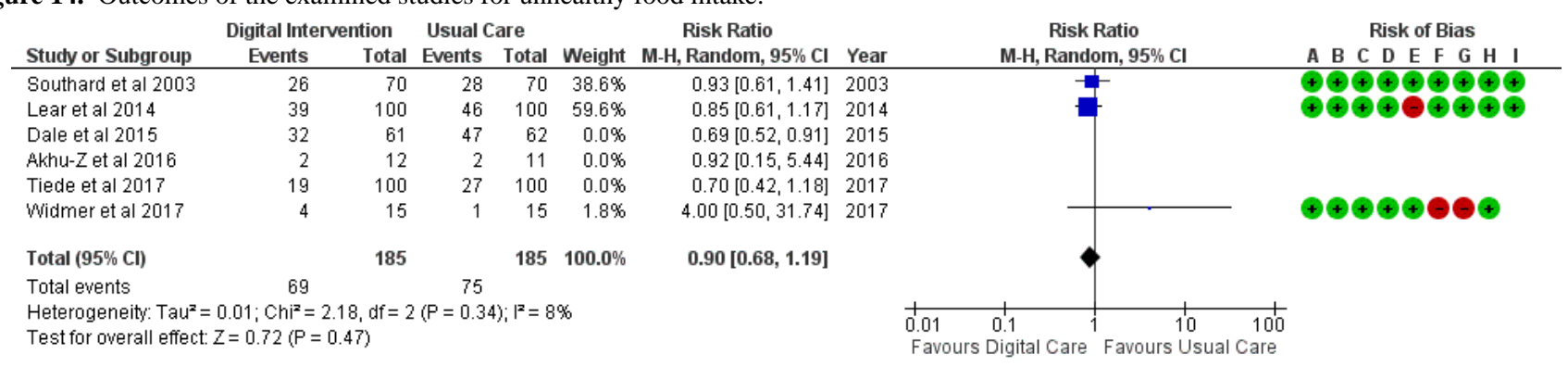

Risk of bias legend

(A) Random sequence generation (selection bias)

(B) Allocation concealment (selection bias)

(C) Blinding of participants and personnel (performance bias)

(D) Blinding of outcome assessment (detection bias)

(E) Incomplete outcome data (attrition bias)

(F) Groups balanced at baseline (proportion bias)

(G) Groups received same intervention (outcome bias)

(H) Selective reporting (reporting bias)

(l) Intention to treat analysis (treatment efficacy)

Figure 15. Outcomes of the examined studies for alcohol consumption.

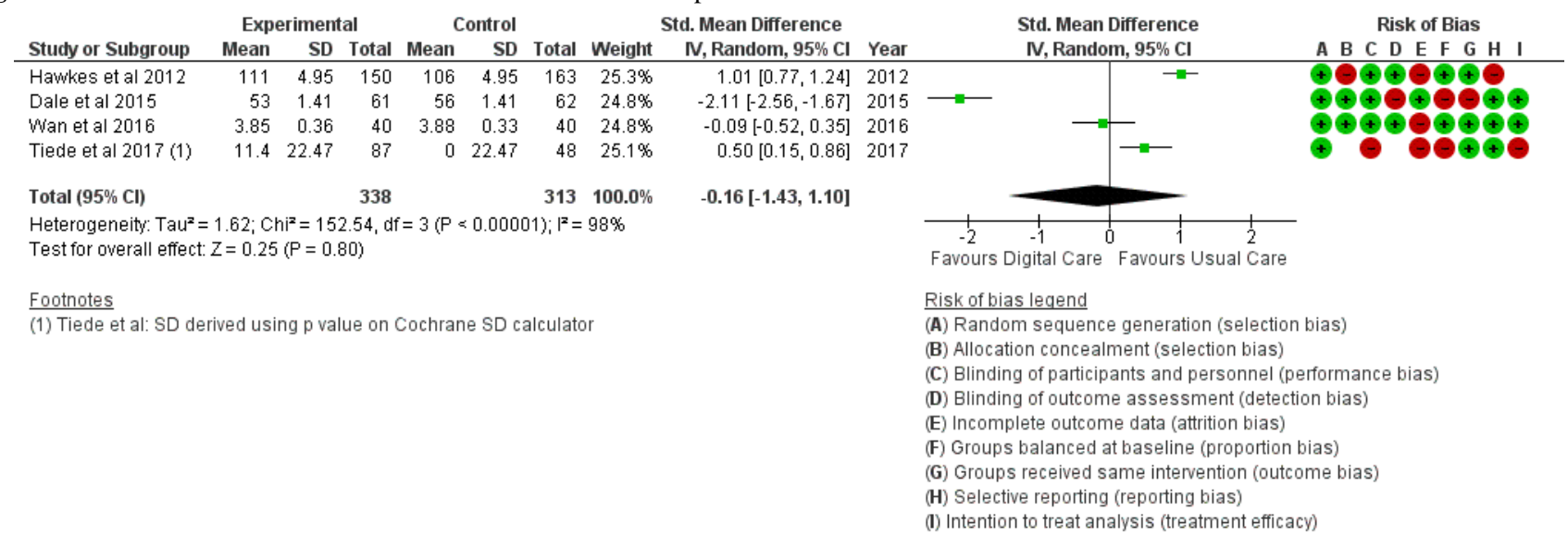

Figure 16. Outcomes of the examined studies for smoking.

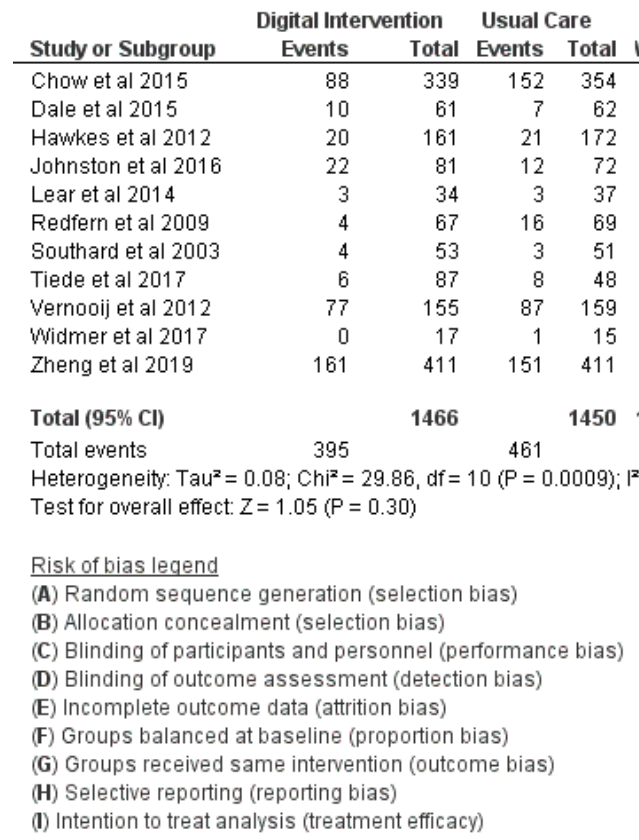

Risk Ratio Risk Ratio

$-\mathrm{H}$, Random, $95 \% \mathrm{Cl} \quad \mathrm{M}-\mathrm{H}$, Random, $95 \% \mathrm{Cl}$

$0.60[0.49,0.75]$

$1.45[0.59,3.57]$

$1.02[0.57,1.81]$

$1.63[0.87,3.05]$

$1.09[0.24,5.03]$

$0.26[0.09,0.73]$

$1.28[0.30,5.45]$

$0.41[0.15,1.12]$

$0.91[0.73,1.12]$

$0.30[0.01,6.77]$

$1.07[0.90,1.27]$

$0.87[0.67,1.13]$

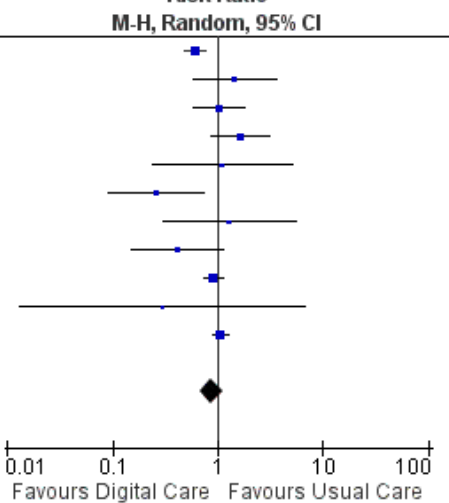

Risk of Bias

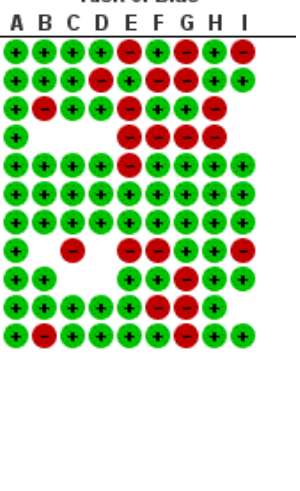


Figure 17. Medication adherence for all trials.

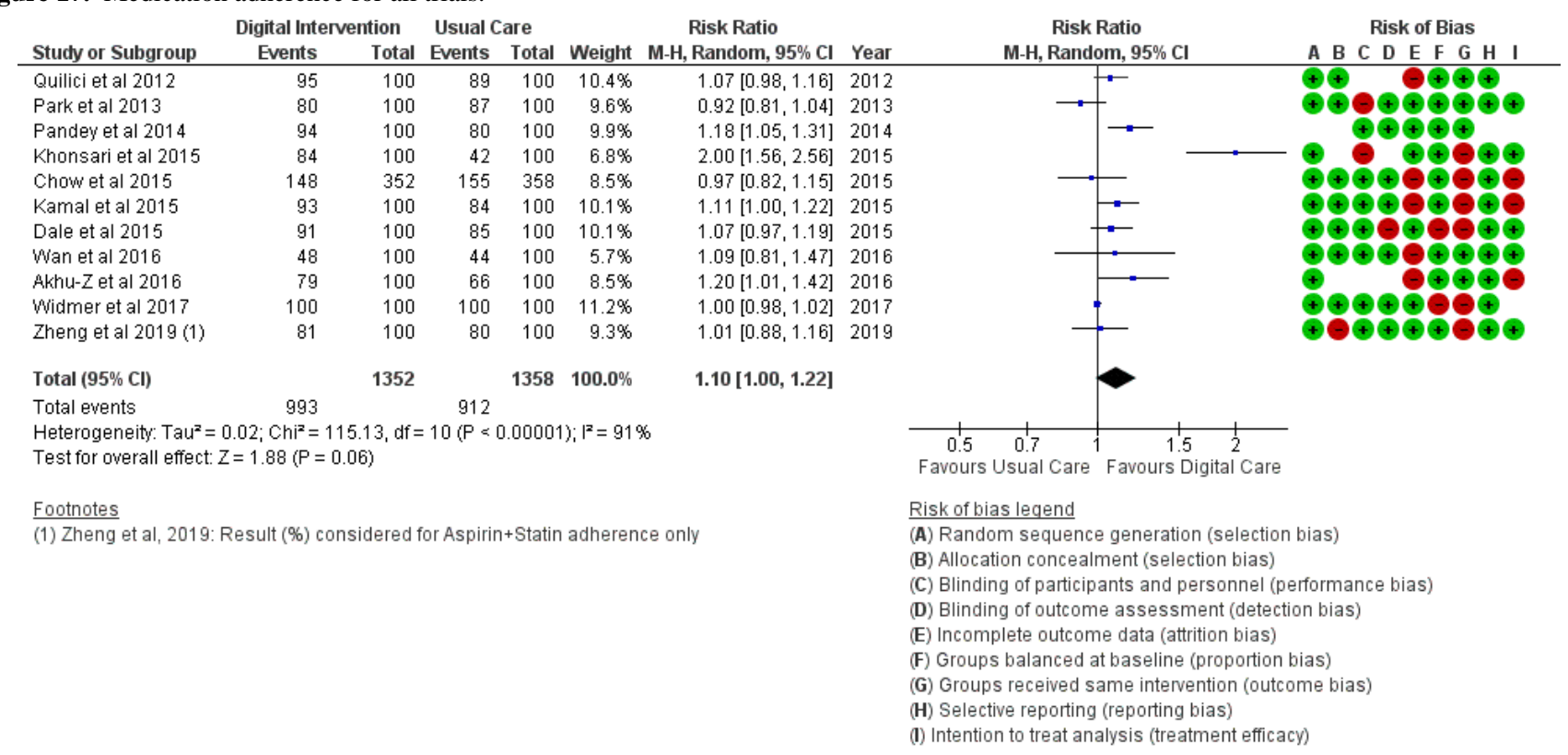

Figure 18. Medication adherence for multiple treatment with SMS.

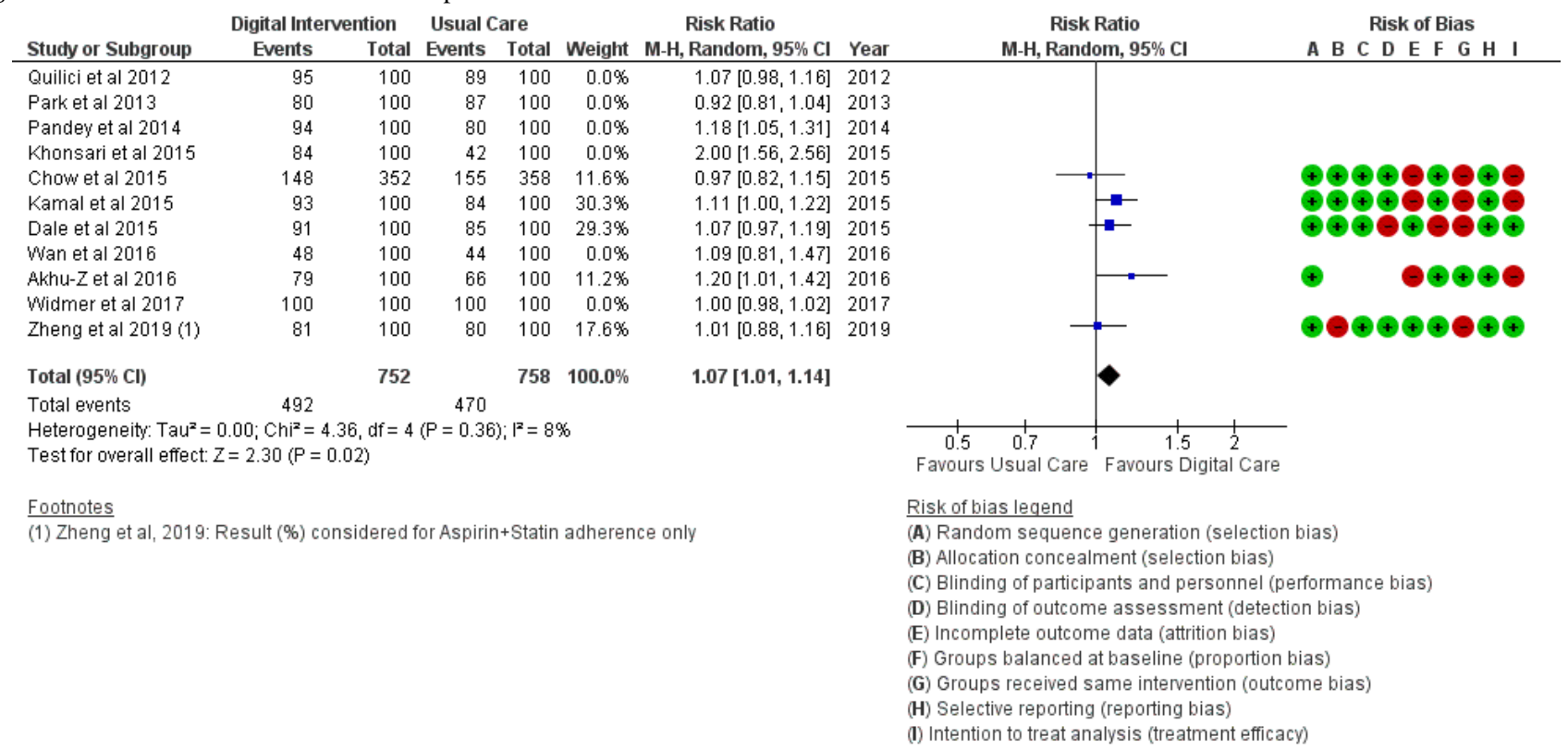


Figure 19. Medication adherence for target treatment only with SMS text message intervention.

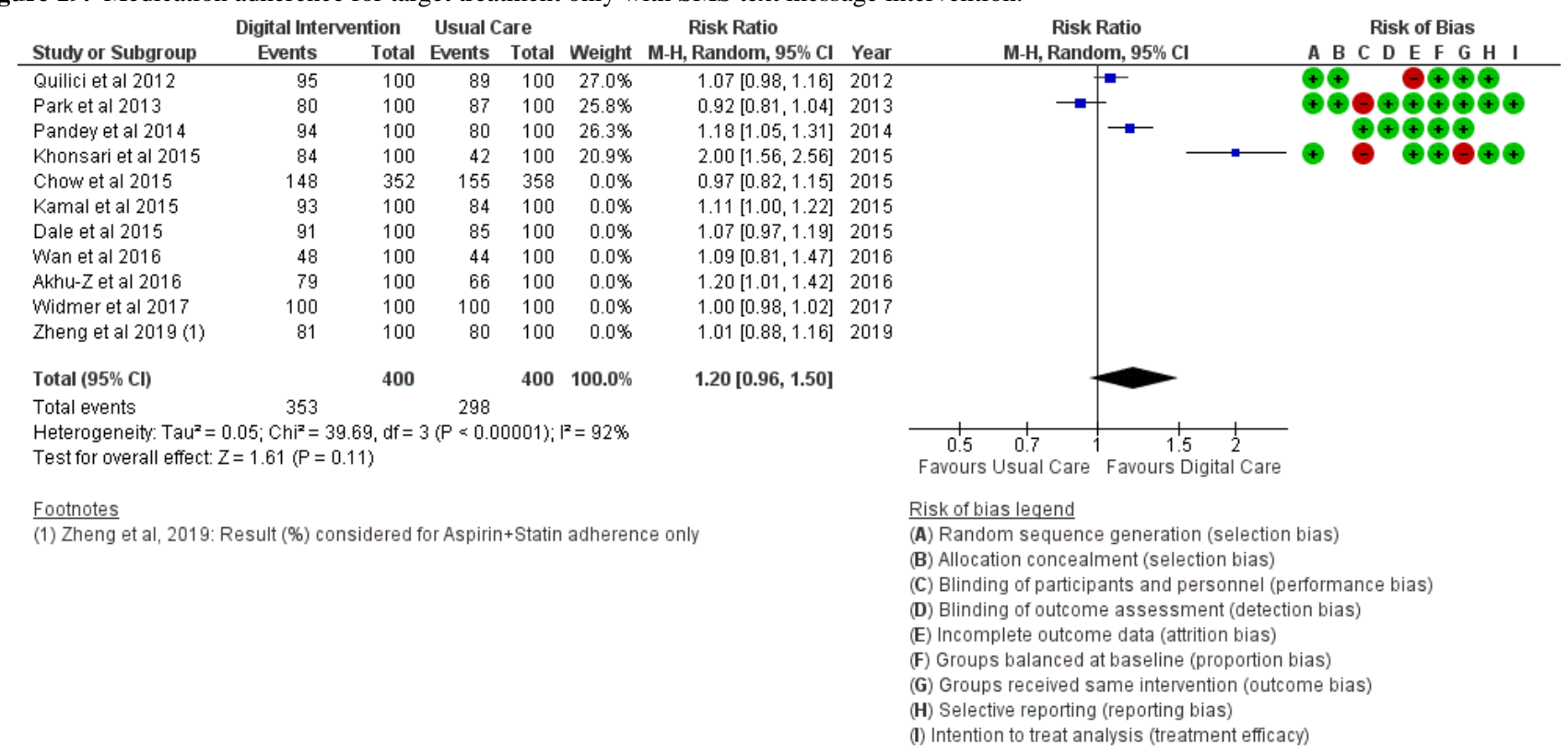

Figure 20. Medication adherence for treatment with non-sms intervention.

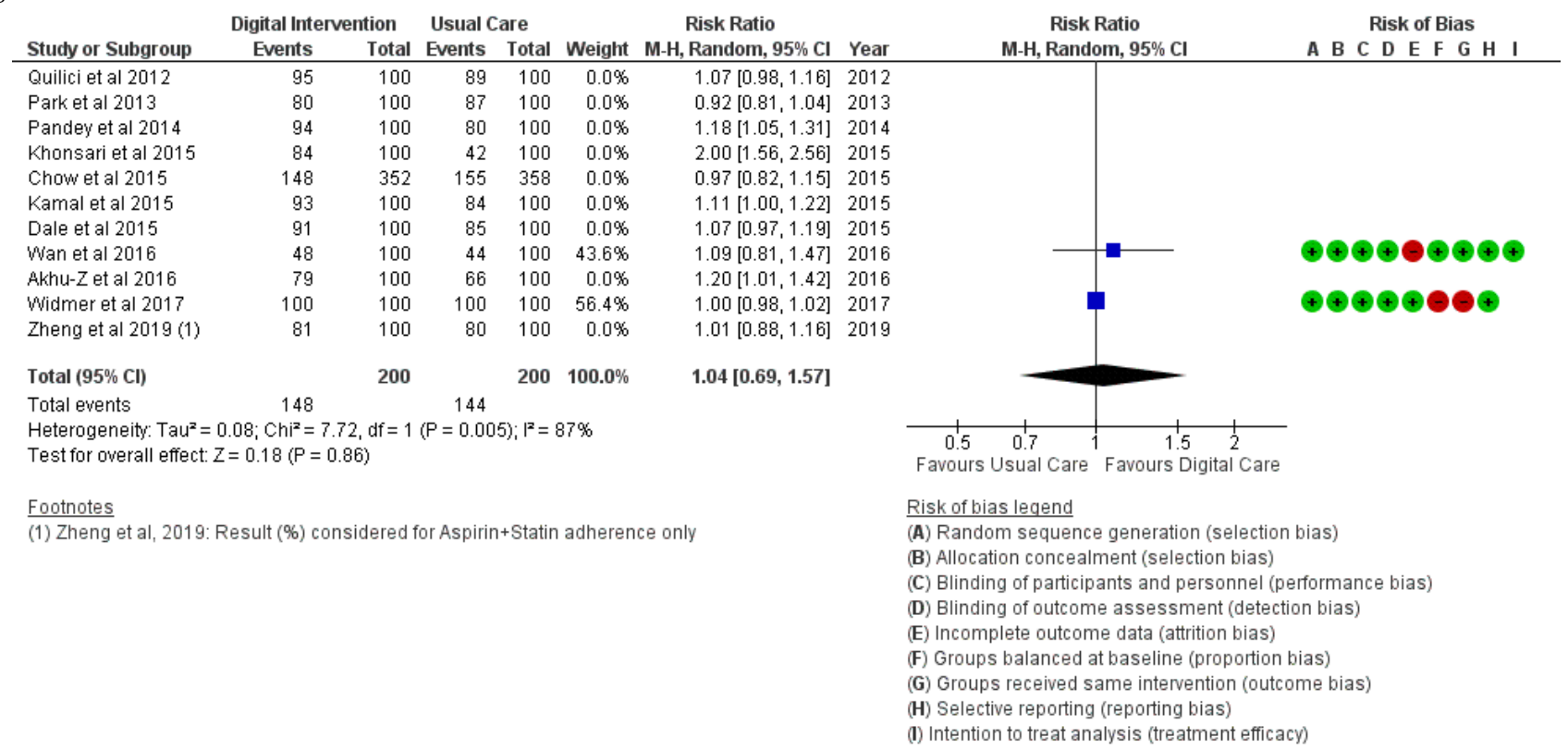


Figure 21. Medication adherence treatment for all sms intervention.

\begin{tabular}{|c|c|c|c|c|c|c|c|c|c|c|}
\hline \multirow[b]{2}{*}{ Study or Subgroup } & \multicolumn{2}{|c|}{ Digital Intervention } & \multicolumn{2}{|c|}{ Usual Care } & \multicolumn{3}{|c|}{ Risk Ratio } & \multirow{2}{*}{\multicolumn{2}{|c|}{$\begin{array}{c}\text { Risk Ratio } \\
\text { M-H, Random, } 95 \% \mathrm{Cl} \\
\end{array}$}} & Risk of Bias \\
\hline & Events & Total & Events & Total & Weight & M-H, Random, $95 \% \mathrm{Cl}$ & Year & & & A B C D E F G H I \\
\hline Quilici et al 2012 & 95 & 100 & 89 & 100 & $13.3 \%$ & $1.07[0.98,1.16]$ & 2012 & & $t=$ & $\odot+\quad \Theta \oplus \odot \odot$ \\
\hline Park et al 2013 & 80 & 100 & 87 & 100 & $11.7 \%$ & $0.92[0.81,1.04]$ & 2013 & & & \\
\hline Pandey et al 2014 & 94 & 100 & 80 & 100 & $12.2 \%$ & $1.18[1.05,1.31]$ & 2014 & & $\because-$ & \\
\hline Khonsari et al 2015 & 84 & 100 & 42 & 100 & $7.1 \%$ & $2.00[1.56,2.56]$ & 2015 & & & \\
\hline Chow et al 2015 & 148 & 352 & 155 & 358 & $9.8 \%$ & $0.97[0.82,1.15]$ & 2015 & & - & \\
\hline Kamal et al 2015 & 93 & 100 & 84 & 100 & $12.6 \%$ & $1.11[1.00,1.22]$ & 2015 & & $=-$ & \\
\hline Dale et al 2015 & 91 & 100 & 85 & 100 & $12.5 \%$ & $1.07[0.97,1.19]$ & 2015 & & $=-$ & \\
\hline Wan et al 2016 & 48 & 100 & 44 & 100 & $0.0 \%$ & $1.09[0.81,1.47]$ & 2016 & & & \\
\hline Akhu- $Z$ et al 2016 & 79 & 100 & 66 & 100 & $9.7 \%$ & $1.20[1.01,1.42]$ & 2016 & & 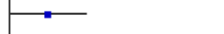 & $\ominus \oplus \odot \odot \ominus$ \\
\hline Widmer et al 2017 & 100 & 100 & 100 & 100 & $0.0 \%$ & $1.00[0.98,1.02]$ & 2017 & & & \\
\hline Zheng et al 2019 (1) & 81 & 100 & 80 & 100 & $11.2 \%$ & $1.01[0.88,1.16]$ & 2019 & & 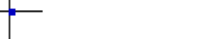 & 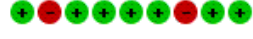 \\
\hline Total $(95 \% \mathrm{Cl})$ & & 1152 & & 1158 & $100.0 \%$ & $1.11[1.01,1.22]$ & & & & \\
\hline \multirow{2}{*}{\multicolumn{8}{|c|}{$\begin{array}{l}\text { Total events } \quad 845 \quad c \quad 768 \\
\text { Heterogeneity: } \text { Tau }^{2}=0.01 ; \mathrm{Ch}^{2}=37.60, \mathrm{df}=8(\mathrm{P}<0.00001) ;\left.\right|^{2}=79 \% \\
\text { Test for overall effect: } Z=2.27(\mathrm{P}=0.02)\end{array}$}} & & & \\
\hline & & & & & & & & $\begin{array}{cc}0.5 & 0.7 \\
\text { Favours Usual Care }\end{array}$ & $1 \frac{1.5}{2}$ & \\
\hline \multicolumn{8}{|c|}{$\frac{\text { Footnotes }}{\text { (1) Zheng et al, 2019: Result (\%) considered for Aspirin+Statin adherence only }}$} & \multicolumn{3}{|c|}{$\begin{array}{l}\text { Risk of bias legend } \\
\text { (A) Random sequence generation (selection bias) } \\
\text { (B) Allocation concealment (selection bias) } \\
\text { (C) Blinding of participants and personnel (performance bias) } \\
\text { (D) Blinding of outcome assessment (detection bias) } \\
\text { (E) Incomplete outcome data (attrition bias) } \\
\text { (F) Groups balanced at baseline (proportion bias) } \\
\text { (G) Groups received same intervention (outcome bias) } \\
\text { (H) Selective reporting (reporting bias) } \\
\text { (I) Intention to treat analysis (treatment efficacy) }\end{array}$} \\
\hline
\end{tabular}

\section{Risk of Bias in Included Studies}

Table 2 provides a qualitative detail of the risk of bias assessment of the studies in the review. Proportion bias at baseline was reported in $16 \%(4 / 25)$ of the included studies as high risk. Intervention dropout was recorded in $32 \%(8 / 25)$ of the included studies at less than $10 \%$ of participants per study. Dropouts greater than $10 \%$ of study participants were recorded as high risk for treatment efficacy.

\section{Risk of Bias Across Studies}

The results of the risk of bias across studies for each outcome are presented along with the meta-analysis (Figures 2-21). Outcomes are sparsely identified with low and unclear risks for the identified risk items.

\section{Additional Analysis}

Results of the sensitivity analysis were included for the considered outcomes in Figures 13 and 14 and Figures 18-21. Sensitivity analyses proportionately conducted on outcomes to check the cumulative effects of the study population and intervention characteristics (eg, publication year, participant size, efficacy, and categories of treatment) on subgroups showed significant effects of interest in 2 outcomes as follows:

Food intake: Healthy diet targeted (Figure 13) treatment $(P=.004)$ and unhealthy diet targeted (Figure 14) treatment $(P=.47)$; medication adherence: medication adherence plus other risk factors (Figure 18) treatment $(P=.02)$ and medication adherence treatment alone $(P=.11)$, as shown in Figure 19.

\section{Discussion}

\section{Principal Findings}

This study of digital technology interventions addressing clinical and behavioral risk factor modification in people with CVDs demonstrates that not all CVD lifestyle risk factor modifications are favored by the use of digital interventions. Digital technology intervention in cardiac patients was associated with improvements in TC, HDL, LDL, PA, PI, healthy diet, and medication adherence (all $P \leq .05$ ). However, there were no differences in intervention effects for BMI, TGs, BP (diastolic and systolic), blood sugar, alcohol intake, and smoking (all $P>$.05).

\section{Behavioral Change Constructs and Digital Intervention Strategies}

The mechanism of risk factor modification in the included studies is based on behavioral change constructs [43,44], which include self-management, feedback mechanisms, progress recording and tracking (monitoring), one-on-one or social support, persuasion, personalization (customization), reiteration, self-efficacy, and motivation. For this study, these constructs are commonly used in the study trials compared with other constructs in the literature, such as perceived risk and perceived benefits, incentives, and reimbursement (rewards), which are rarely used in the study trials.

The use of behavioral change constructs in combination with digital technologies in the study trials has revealed their successful application in individual behavioral risk factor modification [7]. The overall desired effect has been found in digital interventions alone (15 studies) when compared with digital plus usual care interventions (10 studies), giving support to out-of-clinic risk factor modification at the personal level [9].

From our results, the use of behavioral change constructs and digital intervention strategies largely relies on patients' self-dependency (low- to moderate-risk CVD patients), and interventions that favored digital technologies were reported for all CVD populations but barely for study trials on stroke and rarely for study trials on angina outpatients. An exception to this reliance was found in studies on medication adherence, which had been successfully self-managed with a digital intervention (SMS text messaging) - this informed advice-based instead of activity-based options for moderate-risk CVD outpatients in risk factor modification prescriptions. The effect 
of mobile sensor technology using wearable devices was inconclusive as there was only one study that engaged this digital intervention in its trial (Table S4 in Multimedia Appendix $1)$.

\section{Clinical Outcomes}

There was no clinical benefit for BMI, TG, SBP, DBP, or $\mathrm{HbA}_{1 \mathrm{c}}$ with the use of digital interventions compared with usual care intervention in the study trials. This finding suggests a form of association between clinical factors and unhealthy behavior modification using digital technologies, noting that these clinical factors are the main indicators of unhealthy behavior conditions such as obesity, hypercholesterolemia, hypertension, and diabetes.

However, an exception to these similarities is the significant effect of digital technology intervention on clinical factors such as TC, HDL, and LDL, which could only be inferred by their shared physiological response to regulations in and by healthy behavioral factors such as healthy diet and medication adherence from a lifestyle perspective [45]. This response was less impactful on TGs, which are stored lipids in fatty cells and though considered bad cholesterol like LDL, are less regulated by medication (eg, statin) as compared with diet [45]. Our findings suggest that this exception is not necessarily based on the application of behavioral change techniques or other change-effecting variables. This view is validated by the fact that BMI (an indicator of overweight) and $\mathrm{HbA}_{1 \mathrm{c}}$ (an indicator of excess sugar), which are precursors for obesity and diabetes respectively as CVD risk factors, in association with unhealthy food intake appear to be not modifiable by digital technology interventions. Furthermore, we consider that the modification of LDL (bad cholesterol) by digital intervention might have been because of the positive inverse effect derived from the modification benefits of TC and HDL (good cholesterols) within each study population.

TC, HDL, and LDL modifications are associated with the use of cell phone devices in study trials. Behavior change techniques in TC, HDL, and LDL populations include self-reporting and self-recording of progress, one-on-one support, and persuasion. TC, HDL, and LDL study populations share commonly diagnosed CVDs and digital intervention strategies.

\section{Behavioral Outcomes}

PA trials are characterized by smartphones and cell phone devices in a 1:1 ratio. In order of preference, the use of intervention strategies is, first, telerehabilitation and online education, followed by online feedback (tele-support) and telemonitoring, and finally, SMS text messaging support by active coaching ( $20 \%$ of trials). PI (sedentary) trials revealed a higher population mean age, which suggests a close association with comorbidity and immobility among outpatients [46]; therefore, there is a need to tailor digital intervention treatment to patients' level of engagement.

PA and PI have gained modification preferences and digital intervention effectiveness because of active participant engagement in 11 smartphone studies using tele-intervention (audiovisual) strategy, 2 cell phone studies engaging in active coaching (audio) strategy, and 6 cell phone studies using automated SMS text messaging support (text) strategy (in 19 studies). This finding suggests greater effectiveness of smartphones in audiovisual interventions compared with cell phones, indicating that cell phones might have gained usage (in medication adherence study trials) only because of their affordability and ease of use $[44,47]$. However, both audiovisual support and audio or text support appear to be efficient digital interventions for risk factor modification for PA; however, audio or text support only appears sufficient for PI modification.

Generally, PA (a healthy behavioral factor) has been viewed as a null to PI (sedentary; an unhealthy behavioral factor) effects in maintaining a healthy lifestyle. This view has been disapproved in the literature [48]. However, this disapproval has only been validated in a healthy population prospective study. More evidence is needed to validate this in a CVD population to elucidate the effectiveness of digital technology interventions for PI risk factor modification. Therefore, we consider that the modification of PI, just as in LDL, might have been because of the positive inverse effect of PA modification in the CVD populations reviewed.

Studies reporting effects on diet, alcohol consumption, and smoking share similar characteristics in behavioral change techniques, which include mostly social support and group discussion, followed by self-management, goal setting, follow-up, progress self-reporting and self-recording, and auto-reminders. Social support and group discussion, which are related to online support and online discussion, have been identified as activity-based behavioral change techniques in mental health management for diet, alcohol consumption, and smoking behavior modifications [49]. Interactivity (as a result of social support and group discussion) can, therefore, be affirmed as an effective factor in the behavior change technique of diet, alcohol consumption, and smoking on a digital platform. However, digital interventions for alcohol consumption and smoking behavior change show a weak effect in their modification when compared with conventional CVDs' usual care interventions. There could be several reasons for this-first, social support and group discussions or interaction are less effectively accomplished compared with cell phone device interventions, which have no smart facial contact technology features but have gained wider usage in reviewed study trials because of their affordability and availability to participants in both risk factor studies. Second, digital technology interventions, from the trend seen in this study, appear to be effective in healthy behavior modification but less effective in attending to unhealthy behavior modification when compared with usual care: healthy dieting is physiologically linked to lipid regulation in the body [50], a strong basis for clinical factor (TC, HDL, and LDL) modification.

In addition, digital intervention effectiveness in TC, HDL, and LDL, as stated earlier, might also be largely linked to the positive pharmacological effect of medication adherence in study trials. Of the 6 studies on food intake (diet), unhealthy food intake (Figure 14) modification is not favored by digital intervention $(P=.47)$; however, a healthy diet (Figure 13) shows a significant modification effect in favor of digital intervention $(P=.004)$ when compared with usual care. This difference reveals significant alignment and potency of digital intervention 
toward healthy behavioral factors than unhealthy behavioral factors. The same was confirmed for PA, a healthy behavioral factor. Healthy behavioral factor (eg, PA, healthy diet, medication adherence) modification using digital technology is supported by findings from Chow et al [18].

Medication adherence outcome from trials in this study was achieved only by the use of cell phones with SMS text messaging support strategy, in line with the findings of Palmer et al [51]. However, the effectiveness of smartphones is inconclusive, as only 1 trial is available in this study - this could be responsible for its limitation in maximizing change technique features, for example, telerehabilitation in medication adherence trials. Cell phones remain the most affordable and available [47] digital devices in medication adherence-targeted interventions compared with other behavioral factor interventions as they cut across all CVD types and engage behavioral change techniques based on cognition such as auto-support, auto-reminders, persuasion (iteration), goal setting, self-management, and customization (personalization).

Trials (Figure 19) that strictly targeted medication adherence outcome only, using an SMS text messaging strategy with a cell phone device, did not show a significant effect $(P=.11)$ when collectively analyzed for digital intervention effectiveness. However, trials (Figure 18) with a similar strategy and device as the former but having multiple clinical and behavioral outcome treatments (analyzed with or without the previous trials) were significantly effective $(P=.02)$ with the use of digital intervention compared with usual care. Non-SMS-administered medication adherence trials (Figure 20) did not favor digital intervention. In summary, these findings suggest the effectiveness of multiple clinical and behavioral outcome treatments when designing digital technology (SMS text messaging) interventions.

A few meta-analyzed results such as for smoking, LDL, BMI, and SBP were limited by high heterogeneity not fully explained (or not explained at all as for alcohol consumption and sedentary lifestyle with low included study counts) by study population or intervention characteristics. However, minor adjustments (exclusion of Chow et al [18], Widmer et al [8], and Redfern et al [33]) in the number of included studies toward increased homogeneity did not show a significant change from the initial treatment effect by either digital intervention or usual care.

The main intervention strategies in this study are automated SMS text messaging support (auto-reminder based on cognition which is largely accessible using cell phones in study trials), a feature supported by Kassavou et al [44]; and then online education and coaching, followed by telerehabilitation and telemonitoring, which were barely represented in analyses that favored digital intervention-representation might be because of limited access to smartphones based on the participants' affordability or level of technological advancement or inclination at the time of the trial. A desirable device is the smartphone because it combines all operability features needed to attain desirable intervention outcomes by identifying behavioral change-specific strategies. However, a major limitation to the use of smartphones by the population age group in the study could be their level of comprehensibility [5].

\section{Limitations}

Although this collection of studies is evenly distributed on a global scale, no RCT study has been identified in Africa, where only cost-effective digital health programs have presently gained widespread use [47]. A high proportion of male to female patients would be considered a major limitation of participant inclusion in studies. However, this trend appears to be in resonance with quantitative analyses of CVD gender prevalence in the literature [4] and, therefore, may reflect disease prevalence rather than study design.

This study further reveals gaps in the application of emerging technologies (immersive media, eg, 3D animations and games, an ongoing trial by Gallagher et al [52]; big data technologies, eg, artificial intelligence applications; and user experience) in CVD risk factor modification using evidence-based RCT intervention studies on a digital device platform. Therefore, this study suggests the initiation of cutting-edge research in the field of emerging digital technologies.

\section{Conclusions}

This study shows that the use of digital technology interventions did not improve all CVD lifestyle risk factors compared with usual care interventions. Effective digital technology interventions appear to improve healthy behavioral factors (PA, healthy diet) and associated clinical outcomes (TC, HDL, and LDL), and were more potent in multiple outcome treatment (medication adherence plus) but were weak in abating unhealthy behavioral factors (smoking, alcohol intake, and unhealthy food intake) and their outcomes (BMI, BP, and $\mathrm{HbA}_{1 \mathrm{c}}$ ).

Cell phones are considered efficient digital devices for use with cognitive intervention strategies and have been most widely studied; however, smartphones may have advantages because of additional interaction features. This study was not able to analyze cutting-edge technology (such as immersive media technologies) as the data do not exist or are not reported. Newer immersive media technologies, therefore, warrant further study. Further RCT research is deemed necessary to consolidate the use of digital technology interventions, especially in CVD risk factors (eg, diabetes), with fewer RCT studies.

\section{Acknowledgments}

This is a trans-European cardiovascular (ECME) collaboration project, supervised by the University of the Highlands and Islands, UK. This study was supported by the EU's INTERREG VA Program, managed by the Special EU Programs Body (SEUPB).

\section{Conflicts of Interest}

None declared. 


\section{Multimedia Appendix 1}

Protocol registration link and tables and abbreviations and definition of terms.

[DOC File , 469 KB-Multimedia Appendix 1]

\section{Multimedia Appendix 2}

Study characteristics.

[DOC File, 95 KB-Multimedia Appendix 2]

\section{References}

1. Cardiovascular diseases. World Health Organization. 2019. URL: https://www.who.int/health-topics/cardiovascular-diseases/ [accessed 2021-02-11]

2. Hospital Episode Statistics (HES). NHS Digital. 2017. URL: http://www.content.digital.nhs.uk/hes [accessed 2021-02-11]

3. Health diseases. British Heart Foundation. 2014. URL: https://www.bhf.org.uk/old-starts-with-your-heart/heart-diseases [accessed 2021-02-11]

4. Khot UN, Khot MB, Bajzer CT, Sapp SK, Ohman EM, Brener SJ, et al. Prevalence of conventional risk factors in patients with coronary heart disease. J Am Med Assoc 2003 Aug 20;290(7):898-904. [doi: 10.1001/jama.290.7.898] [Medline: 12928466]

5. Rocca HB, Fleischhacker L, Golubnitschaja O, Heemskerk F, Helms T, Hoedemakers T, et al. Challenges in personalised management of chronic diseases-heart failure as prominent example to advance the care process. EPMA J 2015;7:2 [FREE Full text] [doi: 10.1186/s13167-016-0051-9] [Medline: 26913090]

6. Conner CT. Social problems: the limits and strengths of the social constructionist perspective. Symb Interact 2014 Oct 06. [doi: $10.1002 /$ symb.125]

7. Valente TW. Network interventions. Sci 2012 Jul 06;337(6090):49-53. [doi: 10.1126/science.1217330] [Medline: 22767921]

8. Widmer RJ, Collins NM, Collins CS, West CP, Lerman LO, Lerman A. Digital health interventions for the prevention of cardiovascular disease: a systematic review and meta-analysis. Mayo Clin Proc 2015 Apr;90(4):469-480 [FREE Full text] [doi: 10.1016/j.mayocp.2014.12.026] [Medline: 25841251]

9. Franklin NC, Lavie CJ, Arena RA. Personal health technology: a new era in cardiovascular disease prevention. Postgrad Med 2015 Mar;127(2):150-158 [FREE Full text] [doi: 10.1080/00325481.2015.1015396] [Medline: 25690685]

10. Scotland Government. eHealth strategy 2011-2017 (Revised July 2012 to include a Sixth Strategic Aim). In: The eHealth Strategy for NHS Scotland 2011-2017. Scotland: Scotland Government; 2012.

11. Digital technologies improve remote rehabilitation, medical monitoring for patients. Barcelona Digi Cent Tecnològic. 2019. URL: https://www.sciencedaily.com/releases/2013/11/131104035243.htm [accessed 2019-10-13]

12. Krick T, Huter K, Domhoff D, Schmidt A, Rothgang H, Wolf-Ostermann K. Digital technology and nursing care: a scoping review on acceptance, effectiveness and efficiency studies of informal and formal care technologies. BMC Health Serv Res 2019 Jun 20;19(1):400 [FREE Full text] [doi: 10.1186/s12913-019-4238-3] [Medline: 31221133]

13. Charles A, Buck D, Evans H, McKenna H. King's Fund Webcast: what's in store for health and care? King's Fund Webcast, 2019. URL: https://www.kingsfund.org.uk/events/whats-store-health-and-care-2020 [accessed 2021-02-11]

14. Moher D, Liberati A, Tetzlaff J, Altman DG, PRISMA Group. Preferred reporting items for systematic reviews and meta-analyses: the PRISMA statement. PLoS Med 2009 Jul 21;6(7):e1000097 [FREE Full text] [doi: 10.1371/journal.pmed.1000097] [Medline: 19621072]

15. Akinosun AS, Polson R, Grindle M. Digital technologies risk factors modification in patients with cardiovascular disease: a systematic review and meta-analysis. Prospero. 2019. URL: https://www.crd.york.ac.uk/prospero/display record. php?ID=CRD42019139801 [accessed 2021-02-11]

16. World Health Organization, global strategy on digital health 2020-2024 in global strategy on digital health. 2019. URL: https://extranet.who.int/dataform/upload/surveys/183439/files/Draft [accessed 2021-02-11]

17. Akhu-Zaheya LM, Shiyab WY. The effect of short message system (SMS) reminder on adherence to a healthy diet, medication, and cessation of smoking among adult patients with cardiovascular diseases. Int J Med Inform 2017 Feb;98:65-75. [doi: 10.1016/j.ijmedinf.2016.12.003] [Medline: 28034414]

18. Chow CK, Redfern J, Hillis GS, Thakkar J, Santo K, Hackett ML, et al. Effect of lifestyle-focused text messaging on risk factor modification in patients with coronary heart disease: a randomized clinical trial. J Am Med Assoc 2015;314(12):1255-1263. [doi: 10.1001/jama.2015.10945] [Medline: 26393848]

19. Pfaeffli Dale L, Whittaker R, Jiang Y, Stewart R, Rolleston A, Maddison R. Text message and internet support for coronary heart disease self-management: results from the text4heart randomized controlled trial. J Med Internet Res 2015 Oct 21;17(10):e237 [FREE Full text] [doi: 10.2196/jmir.4944] [Medline: 26490012]

20. Devi R, Powell J, Singh S. A web-based program improves physical activity outcomes in a primary care angina population: randomized controlled trial. J Med Internet Res 2014 Sep 12;16(9):e186 [FREE Full text] [doi: 10.2196/jmir.3340] [Medline: 25217464] 
21. Frederix I, Van Driessche N, Hansen D, Berger J, Bonne K, Alders T, et al. Increasing the medium-term clinical benefits of hospital-based cardiac rehabilitation by physical activity telemonitoring in coronary artery disease patients. Eur J Prev Cardiol 2015 Feb;22(2):150-158. [doi: 10.1177/2047487313514018] [Medline: 24249840]

22. Hawkes AL, Patrao TA, Atherton J, Ware RS, Taylor CB, O'Neil A, et al. Effect of a telephone-delivered coronary heart disease secondary prevention program (proactive heart) on quality of life and health behaviours: primary outcomes of a randomised controlled trial. Int J Behav Med 2013 Sep;20(3):413-424. [doi: 10.1007/s12529-012-9250-5] [Medline: 23012159]

23. Johnston N, Bodegard J, Jerström S, Åkesson J, Brorsson H, Alfredsson J, et al. Effects of interactive patient smartphone support app on drug adherence and lifestyle changes in myocardial infarction patients: A randomized study. Am Heart $\mathbf{J}$ 2016 Aug;178:85-94 [FREE Full text] [doi: 10.1016/j.ahj.2016.05.005] [Medline: 27502855]

24. Kamal AK, Shaikh Q, Pasha O, Azam I, Islam M, Memon AA, et al. A randomized controlled behavioral intervention trial to improve medication adherence in adult stroke patients with prescription tailored Short Messaging Service (SMS)-SMS4Stroke study. BMC Neurol 2015 Oct 21;15:212 [FREE Full text] [doi: 10.1186/s12883-015-0471-5] [Medline: 26486857]

25. Khonsari S, Subramanian P, Chinna K, Latif LA, Ling LW, Gholami O. Effect of a reminder system using an automated short message service on medication adherence following acute coronary syndrome. Eur J Cardiovasc Nurs 2015 Apr;14(2):170-179. [doi: 10.1177/1474515114521910] [Medline: 24491349]

26. Kraal JJ, Peek N, Van den Akker-Van Marle ME, Kemps HM. Effects of home-based training with telemonitoring guidance in low to moderate risk patients entering cardiac rehabilitation: short-term results of the FIT@Home study. Eur J Prev Cardiol 2014 Nov;21(2 Suppl):26-31. [doi: 10.1177/2047487314552606] [Medline: 25354951]

27. Lear SA, Singer J, Banner-Lukaris D, Horvat D, Park JE, Bates J, et al. Randomized trial of a virtual cardiac rehabilitation program delivered at a distance via the internet. Circ Cardiovasc Qual Outcomes 2014 Nov;7(6):952-959. [doi: 10.1161/CIRCOUTCOMES.114.001230] [Medline: 25271050]

28. Maddison R, Pfaeffli L, Whittaker R, Stewart R, Kerr A, Jiang Y, et al. A mobile phone intervention increases physical activity in people with cardiovascular disease: results from the HEART randomized controlled trial. Eur J Prev Cardiol 2015 Jun;22(6):701-709. [doi: 10.1177/2047487314535076] [Medline: 24817694]

29. Ögren J, Irewall AL, Söderström L, Mooe T. Long-term, telephone-based follow-up after stroke and TIA improves risk factors: 36-month results from the randomized controlled NAILED stroke risk factor trial. BMC Neurol 2018 Sep 21;18(1):153 [FREE Full text] [doi: 10.1186/s12883-018-1158-5] [Medline: 30241499]

30. Pandey A, Choudhry N. Text message reminders to address medication non-adherence in post-mi patients: a one year intervention study. Can J Cardiol 2014 Oct;30(10):S179. [doi: 10.1016/j.cjca.2014.07.280]

31. Park LG, Howie-Esquivel J, Chung ML, Dracup K. A text messaging intervention to promote medication adherence for patients with coronary heart disease: a randomized controlled trial. Patient Educ Couns 2014 Feb;94(2):261-268. [doi: 10.1016/j.pec.2013.10.027] [Medline: 24321403]

32. Quilici J, Fugon L, Beguin S, Morange PE, Bonnet JL, Alessi MC, et al. Effect of motivational mobile phone short message service on aspirin adherence after coronary stenting for acute coronary syndrome. Int J Cardiol 2013 Sep 20;168(1):568-569. [doi: 10.1016/j.ijcard.2013.01.252] [Medline: 23462636]

33. Redfern J, Briffa T, Ellis E, Freedman SB. Choice of secondary prevention improves risk factors after acute coronary syndrome: 1-year follow-up of the CHOICE (Choice of Health Options In prevention of Cardiovascular Events) randomised controlled trial. Heart 2009 Mar;95(6):468-475. [doi: 10.1136/hrt.2008.150870] [Medline: 18801781]

34. Reid RD, Morrin LI, Beaton LJ, Papadakis S, Kocourek J, McDonnell L, et al. Randomized trial of an internet-based computer-tailored expert system for physical activity in patients with heart disease. Eur J Prev Cardiol 2012 Dec;19(6):1357-1364. [doi: 10.1177/1741826711422988] [Medline: 21903744]

35. Southard BH, Southard DR, Nuckolls J. Clinical trial of an internet-based case management system for secondary prevention of heart disease. J Cardiopulm Rehabil 2003;23(5):341-348. [doi: 10.1097/00008483-200309000-00003] [Medline: 14512778]

36. Tiede M, Dwinger S, Herbarth L, Härter M, Dirmaier J. Long-term effectiveness of telephone-based health coaching for heart failure patients: a post-only randomised controlled trial. J Telemed Telecare 2017 Sep;23(8):716-724. [doi: 10.1177/1357633X16668436] [Medline: 27605214]

37. Vale MJ, Jelinek MV, Best JD, Santamaria JD. Coaching patients with coronary heart disease to achieve the target cholesterol: a method to bridge the gap between evidence-based medicine and the "real world"--randomized controlled trial. J Clin Epidemiol 2002 Mar;55(3):245-252. [doi: 10.1016/s0895-4356(01)00460-7] [Medline: 11864795]

38. Vernooij JW, Kaasjager HA, van der Graaf Y, Wierdsma J, Grandjean HM, Hovens MM, SMARTStudy Group. Internet based vascular risk factor management for patients with clinically manifest vascular disease: randomised controlled trial. BMJ 2012 Jun 12;344:e3750 [FREE Full text] [doi: 10.1136/bmj.e3750] [Medline: 22692651]

39. Wan LH, Zhang XP, Mo MM, Xiong XN, Ou CL, You LM, et al. Effectiveness of goal-setting telephone follow-up on health behaviors of patients with ischemic stroke: a randomized controlled trial. J Stroke Cerebrovasc Dis 2016 Sep;25(9):2259-2270. [doi: 10.1016/j.jstrokecerebrovasdis.2016.05.010] [Medline: 27371106] 
40. Widmer RJ, Allison TG, Lennon R, Lopez-Jimenez F, Lerman LO, Lerman A. Digital health intervention during cardiac rehabilitation: a randomized controlled trial. Am Heart J 2017 Jun;188:65-72. [doi: 10.1016/j.ahj.2017.02.016] [Medline: 28577682]

41. Zheng X, Spatz ES, Bai X, Huo X, Ding Q, Horak P, et al. Effect of text messaging on risk factor management in patients with coronary heart disease: the CHAT randomized clinical trial. Circ Cardiovasc Qual Outcomes 2019 Apr;12(4):e005616. [doi: 10.1161/CIRCOUTCOMES.119.005616] [Medline: $\underline{\text { 30998400] }}$

42. Kraal JJ, Peek N, van den Akker-Van Marle ME, Kemps HMC. Effects and costs of home-based training with telemonitoring guidance in low to moderate risk patients entering cardiac rehabilitation: the FIT@Home study. BMC Cardiovasc Disord 2013 Oct 08;13:82 [FREE Full text] [doi: 10.1186/1471-2261-13-82] [Medline: 24103384]

43. Vale MJ, Jelinek MV, Best JD, Santamaria JD. Coaching patients with coronary heart disease to achieve the target cholesterol: a method to bridge the gap between evidence-based medicine and the. J Clin Epidemiol 2002 Mar;55(3):245-252. [doi: 10.1016/s0895-4356(01)00460-7] [Medline: 11864795]

44. Kassavou A, Houghton V, Edwards S, Brimicombe J, Sutton S. Development and piloting of a highly tailored digital intervention to support adherence to antihypertensive medications as an adjunct to primary care consultations. BMJ Open 2019 Jan 06;9(1):e024121 [FREE Full text] [doi: 10.1136/bmjopen-2018-024121] [Medline: $\underline{30613027}$ ]

45. Lower your cholesterol. NHS. 2019. URL: https://www.nhs.uk/live-well/healthy-body/lower-your-cholesterol/ [accessed 2019-10-18]

46. Figueiró TH, Arins GCB, Santos CESD, Cembranel F, Medeiros PAD, d'Orsi E, et al. Association of objectively measured sedentary behavior and physical activity with cardiometabolic risk markers in older adults. PLoS One 2019 Jan 18;14(1):e0210861 [FREE Full text] [doi: 10.1371/journal.pone.0210861] [Medline: $\underline{\text { 30657795] }}$

47. Healthcare and economic growth in Africa: digital health. UNECA, GBCHealth and Aliko Dangote Foundation. 2019. URL: http://gbchealth.org/healthcare-and-economic-growth-in-africa-digital-health/ [accessed 2021-02-11]

48. Katzmarzyk PT. Physical activity, sedentary behavior, and health: paradigm paralysis or paradigm shift? Diabetes 2010 Nov;59(11):2717-2725 [FREE Full text] [doi: 10.2337/db10-0822] [Medline: 20980470]

49. National Institute for Health and Care Excellence. Behaviour change: digital and mobile health interventions. NICE. 2020. URL: https://www.nice.org.uk/guidance/gid-ng10101/documents/final-scope [accessed 2021-02-11]

50. Udenigwe CC, Rouvinen-Watt K. The role of food peptides in lipid metabolism during dyslipidemia and associated health conditions. Int J Mol Sci 2015 Apr 24;16(5):9303-9313 [FREE Full text] [doi: 10.3390/ijms16059303] [Medline: 25918936]

51. Palmer MJ, Barnard S, Perel P, Free C. Mobile phone-based interventions for improving adherence to medication prescribed for the primary prevention of cardiovascular disease in adults. Cochrane Database Syst Rev 2018 Jun 22;6:CD012675 [FREE Full text] [doi: 10.1002/14651858.CD012675.pub2] [Medline: 29932455]

52. Gallagher R, Chow C, Parker H, Neubeck L, Celermajer D, Redfern J, et al. Design and rationale of the MyHeartMate study: a randomised controlled trial of a game-based app to promote behaviour change in patients with cardiovascular disease. BMJ Open 2019 May 14;9(5):e024269 [FREE Full text] [doi: 10.1136/bmjopen-2018-024269] [Medline: 31092643 ]

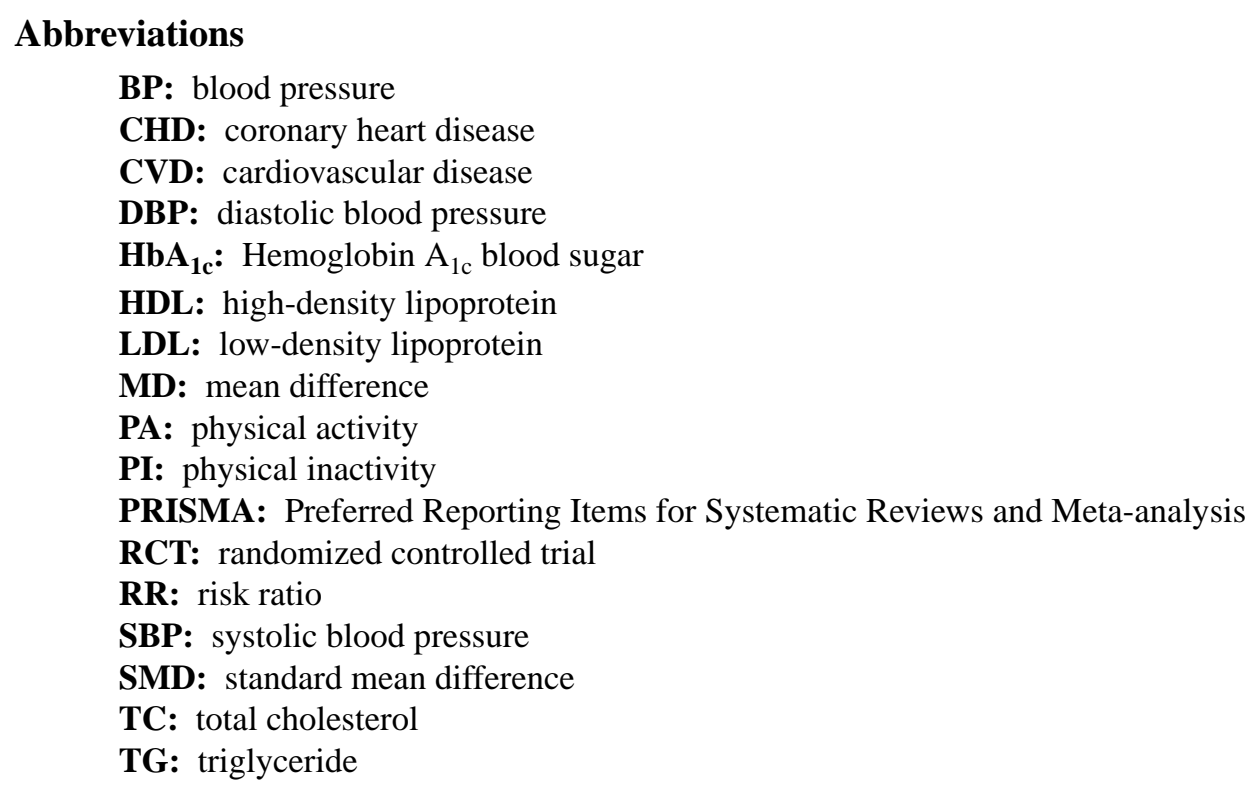


Edited by L Buis; submitted 05.06.20; peer-reviewed by L Park, $R$ Lundin, J Li, J Van den Eynde, N Mohammad Gholi Mezerji; comments to author 14.09.20; revised version received 08.11.20; accepted 01.12.20; published 03.03.21

Please cite as:

Akinosun AS, Polson R, Diaz - Skeete Y, De Kock JH, Carragher L, Leslie S, Grindle M, Gorely T

Digital Technology Interventions for Risk Factor Modification in Patients With Cardiovascular Disease: Systematic Review and Meta-analysis

JMIR Mhealth Uhealth 2021;9(3):e21061

URL: https://mhealth.jmir.org/2021/3/e21061

doi: $\underline{10.2196 / 21061}$

PMID: $\underline{36566444}$

(C)Adewale Samuel Akinosun, Rob Polson, Yohanca Diaz - Skeete, Johannes Hendrikus De Kock, Lucia Carragher, Stephen Leslie, Mark Grindle, Trish Gorely. Originally published in JMIR mHealth and uHealth (http://mhealth.jmir.org), 03.03.2021. This is an open-access article distributed under the terms of the Creative Commons Attribution License (https://creativecommons.org/licenses/by/4.0/), which permits unrestricted use, distribution, and reproduction in any medium, provided the original work, first published in JMIR mHealth and uHealth, is properly cited. The complete bibliographic information, a link to the original publication on http://mhealth.jmir.org/, as well as this copyright and license information must be included. 